TRANSACTIONS OF THE

AMERICAN MATHEMATICAL SOCIETY

Volume 361, Number 11, November 2009, Pages 5983-6017

S 0002-9947(09)04785-0

Article electronically published on June 9, 2009

\title{
ON THE LONG TIME BEHAVIOR OF SECOND ORDER DIFFERENTIAL EQUATIONS WITH ASYMPTOTICALLY SMALL DISSIPATION
}

\author{
ALEXANDRE CABOT, HANS ENGLER, AND SÉBASTIEN GADAT
}

\begin{abstract}
We investigate the asymptotic properties as $t \rightarrow \infty$ of the following differential equation in the Hilbert space $H$ :

$$
\ddot{x}(t)+a(t) \dot{x}(t)+\nabla G(x(t))=0, \quad t \geq 0,
$$

where the map $a: \mathbb{R}_{+} \rightarrow \mathbb{R}_{+}$is nonincreasing and the potential $G: H \rightarrow \mathbb{R}$ is of class $\mathcal{C}^{1}$. If the coefficient $a(t)$ is constant and positive, we recover the so-called "Heavy Ball with Friction" system. On the other hand, when $a(t)=1 /(t+1)$ we obtain the trajectories associated to some averaged gradient system. Our analysis is mainly based on the existence of some suitable energy function. When the function $G$ is convex, the condition $\int_{0}^{\infty} a(t) d t=\infty$ guarantees that the energy function converges toward its minimum. The more stringent condition $\int_{0}^{\infty} e^{-\int_{0}^{t} a(s) d s} d t<\infty$ is necessary to obtain the convergence of the trajectories of $(\mathcal{S})$ toward some minimum point of $G$. In the one-dimensional setting, a precise description of the convergence of solutions is given for a general nonconvex function $G$. We show that in this case the set of initial conditions for which solutions converge to a local minimum is open and dense.
\end{abstract}

\section{INTRODUCTION}

Throughout this paper, we study the differential equation

$$
\ddot{x}(t)+a(t) \dot{x}(t)+\nabla G(x(t))=0, \quad t \geq 0
$$

in a finite- or infinite-dimensional Hilbert space $H$, where the map $G: H \rightarrow \mathbb{R}$ is at least of class $\mathcal{C}^{1}$ and $a: \mathbb{R}_{+} \rightarrow \mathbb{R}_{+}$is a nonincreasing function. To motivate our study, let us describe four examples and applications which are intimately connected with equation $(\mathcal{S})$.

Averaged gradient system. For the potential $G$, the much studied gradient flow is defined as the solution map $y(0) \mapsto y(s), s \geq 0$ of the differential equation

$$
\dot{y}(s)=-g(y(s))=-\nabla G(y(s)) .
$$

It is of interest to consider the case where $\dot{y}(s)$ is proportional, not to the instantaneous value of $\nabla G(y(s))$, but to some average of $\nabla G(y(\tau)), \tau \leq s$. The simplest such equation is

$$
\dot{z}(s)+\frac{1}{s} \int_{0}^{s} g(z(\tau)) d \tau=0
$$

Received by the editors October 22, 2007.

2000 Mathematics Subject Classification. Primary 34G20, 34A12, 34D05.

Key words and phrases. Differential equation, dissipative dynamical system, vanishing damping, averaged gradient system, asymptotic behavior, Bessel equation.

(C)2009 American Mathematical Society Reverts to public domain 28 years from publication 
For more general gradient systems with memory terms involving kernels, we refer for example to 9]. After multiplying equation (11) by $s$ and differentiating, this leads to the ordinary differential equation

$$
s \ddot{z}(s)+\dot{z}(s)+g(z(s))=0,
$$

which becomes

$$
\ddot{x}(t)+\frac{1}{t} \dot{x}(t)+g(x(t))=0
$$

after the change of variables $s=\frac{t^{2}}{4}, t=2 \sqrt{s}, x(t)=z\left(\frac{t^{2}}{4}\right), z(s)=x(2 \sqrt{s})$. This is the problem $(\mathcal{S})$ with $a(t)=\frac{1}{t}$. We note that in the special case where $g(\xi)=\xi$, (21) is a Bessel equation. All solutions with a finite limit at $t=0$ are multiples of

$$
J_{0}(t)=\sum_{k=0}^{\infty}(-1)^{k} \frac{t^{2 k}}{2^{2 k}(k !)^{2}} .
$$

It is well known that

and therefore

$$
J_{0}(t) \sim \sqrt{\frac{2}{\pi t}} \cos \left(t-\frac{\pi}{4}\right)
$$

$$
z(s)=C \cdot J_{0}(2 \sqrt{s}) \sim C \cdot \sqrt[4]{\frac{1}{s}} \cos \left(2 \sqrt{s}-\frac{\pi}{4}\right)
$$

for some suitable constant $C$ as $s, t \rightarrow \infty$. Thus the solution $z$ of the averaged system (11) converges to zero just as the solution $y(s)=y(0) e^{-s}$ of the corresponding gradient system does, but it does so much more slowly (at an algebraic rate), and it oscillates infinitely often. Our work will generalize this simple famous example using several cases for $a$ and $G$. The case where $g(x)=x^{3}-x$ and $H=\mathbb{R}$ was discussed in 15.

Heavy Ball with Friction System. Particular attention has recently been devoted to the so-called "Heavy Ball with Friction" system

$$
\ddot{x}(t)+\gamma \dot{x}(t)+\nabla G(x(t))=0,
$$

where $\gamma>0$ is a positive damping parameter. From a mechanical point of view, the $(H B F)$ system corresponds to the equation describing the motion of a material point subjected to the conservative force $-\nabla G(x)$ and the viscous friction force $-\gamma \dot{x}$.

The $(H B F)$ system is dissipative and can be studied in the classical framework of the theory of dissipative dynamical systems ( $c f$. Hale [10, Haraux [11]). The presence of the inertial term $\ddot{x}(t)$ allows us to overcome some drawbacks of the steepest descent method. The main interest of the $(H B F)$ system in numerical optimization is that it is not a descent method: it permits going up and down along the graph of $G$. The trajectories of $(H B F)$ are known to be convergent toward a critical point of $G$ under various assumptions such as convexity, analyticity, etc. In the convex setting, the proof of convergence relies on the Opial lemma, see Alvarez [2], Attouch-Goudou-Redont [5], while it uses the Lojasiewicz inequality in the case of analytic assumptions ( $c f$. Haraux-Jendoubi [12]).

In the above $(H B F)$ model, the damping coefficient $\gamma$ is constant. A natural extension consists in introducing a time-dependent damping coefficient, thus leading to the system $(\mathcal{S})$. In our paper, we will focus on the important case corresponding to a vanishing damping term $a(t)$, i.e. $a(t) \rightarrow 0$ as $t \rightarrow \infty$. It is clear that the decay 
properties of the map a play a central role in the asymptotic behavior of $(\mathcal{S})$. In particular, if the quantity $a(t)$ tends to 0 too rapidly as $t \rightarrow \infty$, convergence of the trajectory may fail (think about the extreme case of $a \equiv 0$ for instance).

Semilinear elliptic equations. Consider the semilinear elliptic system

$$
\Delta u(y)+g(u(y))=0
$$

in $\mathbb{R}^{m}$, where $u: \mathbb{R}^{m} \rightarrow \mathbb{R}^{n}$ is the unknown function. Radial solutions $u(y)=x(|y|)$ of this system lead to the ordinary differential equation,

$$
\ddot{x}(r)+\frac{m-1}{r} \dot{x}(r)+g(x(r))=0 .
$$

There has been a large amount of work on this problem; see e.g. 16] for a recent overview.

Stochastic approximation algorithms. The classical stochastic algorithm introduced by [17] is used in many fields of approximation theory. This method is frequently used to approximate, with a random version of the explicit Euler scheme, the behavior of the ordinary differential equation $\dot{x}(t)=-g(x(t))$. If we denote by $\left(X^{n}\right)_{n \in \mathbb{N}}$ the random approximations and by $\left(\omega^{n}\right)_{n \geq 1}$ and $\left(\eta^{n}\right)_{n \geq 1}$ two auxiliary stochastic processes, the recursive approximation is generally written as

$$
\left\{\begin{array}{l}
X^{0} \in \mathbb{R}^{d} \\
X^{n+1}=X^{n}-\varepsilon_{n+1} g\left(X^{n}, \omega^{n+1}\right)+\varepsilon_{n+1} \eta^{n+1}, \quad \forall t \in \mathbb{N},
\end{array}\right.
$$

where the gain of the algorithm $\varepsilon_{n}$ is a sequence of positive real numbers and $\eta^{n}$ is a small residual perturbation which is zero in many cases. Defining by $\left(\mathcal{F}_{n}\right)_{n \geq 1}$ the set of measurable events at time $t=n$, solutions of (3) are shown to asymptotically behave like those of the determinist o.d.e. $\dot{x}(t)=-g(x(t))$ provided $\eta^{n}=o\left(\varepsilon^{n}\right)$, $\Delta M^{n}=g\left(X^{n}\right)-g\left(X^{n}, \omega^{n+1}\right)$ is the increment of a (local) $\mathcal{F}_{n}$-martingale, and the sequence $\left(\varepsilon_{n}\right)_{n \geq 1}$ satisfies the baseline assumptions:

$$
\sum_{n=1}^{\infty} \varepsilon_{n}=\infty \quad \text { and } \quad \sum_{n=1}^{\infty} \varepsilon_{n}^{1+\alpha}<\infty, \quad \text { for some } \alpha>0 .
$$

The assumption on the martingale increment implies that

$$
g\left(X^{n}\right)=\mathbb{E}\left[g\left(X^{n}, \omega^{n+1}\right) \mid \mathcal{F}^{n}\right] .
$$

A very common case occurs when $\left(\omega^{n}\right)_{n \geq 1}$ is a sequence of independent identically distributed variables with distribution $\mu$ and $g(x,$.$) is \mu$-integrable: $g(x):=$ $\int g(x, \omega) \mu(d \omega)$. This yields the stochastic gradient descent algorithm when $g$ is the gradient operator of a potential $G$. One recent work 8 applied this stochastic gradient to perform feature selection among a large number of variables with a simpler Euler scheme $X^{n+1}=X^{n}-\varepsilon_{n} g\left(X^{n}, \omega^{n+1}\right)$. Further developments have shown that, in some cases, the random variable $g\left(X^{n}\right.$, .) may have a large variance and the stochastic approximation of $g\left(X^{n}\right)$ by $g\left(X^{n}, \omega^{n+1}\right)$ can be numerically improved using the following modified recursive definition:

$$
X^{n+1}=X^{n}-\varepsilon_{n+1} \frac{\sum_{i=1}^{n} \varepsilon_{i} g\left(X^{i}, \omega^{i+1}\right)}{\sum_{i=1}^{n} \varepsilon_{i}} .
$$


One can think about (4) as a way to improve the stability of the gradient estimate $g\left(X^{n}\right)$ by an average on the variables $\left\{g\left(X^{k}, \omega^{k+1}\right), k \leq n\right\}$ whose weights correspond to the $\varepsilon_{n}$. Actually, one can show (see the proof in Appendix A) that the limit o.d.e. is given by an equation of type $(\mathcal{S})$ :

$$
\ddot{X}(t)=-\frac{\dot{X}(t)+g(X(t))}{t+\beta},
$$

for some $\beta \geq 0$. In the particular case $\beta=0$, we obtain the average gradient system equation (11).

The analysis of the asymptotic behavior of $(\mathcal{S})$ is based on the use of the energy function $\mathcal{E}$ defined by $\mathcal{E}(t)=\frac{1}{2}|\dot{x}(t)|^{2}+G(x(t))$ for every $t \geq 0$. Under convex-like assumptions on $G$, we prove the convergence of the quantity $\mathcal{E}(t)$ toward $\min G$ as $t \rightarrow \infty$, provided that $\int_{0}^{\infty} a(t) d t=\infty$. This condition expresses that the damping coefficient $a(t)$ slowly tends to 0 as $t \rightarrow \infty$. Such a condition has already been pointed out for the steepest descent method combined with Tikhonov viscosity-regularization in convex minimization [3] as well as for the stabilization of nonlinear oscillators [4, 7]. When the convex function $G$ has a unique minimum $\bar{x}$, condition $\int_{0}^{\infty} a(t) d t=\infty$ is sufficient to ensure the convergence of the trajectories of $(\mathcal{S})$ toward $\bar{x}$. If the function $G$ has a set of nonisolated equilibria, the more stringent condition $\int_{0}^{\infty} e^{-\int_{0}^{t} a(s) d s} d t<\infty$ is necessary to obtain the convergence of the trajectories of $(\mathcal{S})$ toward some minimum point of $G$. Notice that the previous condition fails if $a(t)=\frac{1}{t+1}$ for every $t \geq 0$, which shows that the averaged gradient system defined above is divergent when the convex function $G$ has multiple minima.

We also have substantial results in the nonconvex setting, when the function $G$ has finitely many critical points. Under the slow condition $\int_{0}^{\infty} a(t) d t=\infty$, we then prove that the energy function $\mathcal{E}(t)$ converges toward a critical value as $t \rightarrow \infty$. If moreover there exists $c>0$ such that $a(t) \geq \frac{c}{t+1}$ for every $t \geq 0$, we show that a Cesaro average of the solution $x$ converges toward some critical point of $G$. Finally, in the one-dimensional setting, a precise description of the convergence of solutions is given for a general nonconvex function $G$. We show that in this case the set of initial conditions for which solutions converge to a local minimum is open and dense.

Outline of the paper. Our work starts with a global existence result of solutions to $(\mathcal{S})$, based on the use of the Lyapounov function $\mathcal{E}$. Section 3 is concerned with the asymptotic behavior of the energy function $\mathcal{E}$ under convex-like hypotheses on $G$, and provides estimates on the speed of convergence of the quantity $\mathcal{E}(t)$ toward $\inf G$ as $t \rightarrow \infty$. Section 4 explores the convergence of the trajectories of $(\mathcal{S})$ in the general setting of convex functions having multiple minima. In section 5 we study the asymptotic behavior of $(\mathcal{S})$ in the nonconvex case when $G$ has finitely many critical points. Finally section 6 is dedicated to the very special one-dimensional case. Details for the stochastic gradient descent algorithm are given in Appendix A, and some special equations are discussed in Appendix B.

\section{General facts}

In the entire paper, we will denote by $G$ a $\mathcal{C}^{1}$ potential map from a Hilbert space $H$ into $\mathbb{R}$ for which the gradient $g=\nabla G$ is Lipschitz continuous, uniformly on bounded sets. Given a function $a: \mathbb{R}_{+} \rightarrow \mathbb{R}_{+}$, we will consider the following 
dynamical system:

$$
\ddot{x}(t)+a(t) \dot{x}(t)+g(x(t))=0, \quad t \geq 0 .
$$

Let us start with a basic result on existence and uniqueness for solutions of $(\mathcal{S})$. In the next statement the map $a$ may have a singularity at $t=0$ so as to cover cases such as $a(t)=1 / t$, for $t>0$.

Proposition 2.1. (a) Suppose $a:(0, \infty) \rightarrow \mathbb{R}_{+}$is continuous on $(0, \infty)$ and integrable on $(0,1)$. Then for any $\left(x_{0}, x_{1}\right) \in H \times H$, there exists a unique solution $x(\cdot) \in \mathcal{C}^{2}([0, T), H)$ of $(\mathcal{S})$ satisfying $x(0)=x_{0}, \dot{x}(0)=x_{1}$ on some maximal time interval $[0, T) \subset[0, \infty)$.

(b) Suppose $a:(0, \infty) \rightarrow \mathbb{R}_{+}$is continuous and there exists $c>0$ such that $a(t) \leq \frac{c}{t}$ for $t \in(0,1]$. Then for any $x_{0} \in H$, there exists a unique solution $x(\cdot) \in \mathcal{C}^{2}((0, T), H) \cap \mathcal{C}^{1}([0, T), H)$ of $(\mathcal{S})$ satisfying $x(0)=x_{0}, \dot{x}(0)=0$ on some maximal time interval $[0, T) \subset[0, \infty)$.

The previous proposition can be proved with standard arguments for ordinary differential equations. The result below states the decay property of the energy function $\mathcal{E}$ defined by

$$
\mathcal{E}(t)=\frac{1}{2}|\dot{x}(t)|^{2}+G(x(t)) .
$$

A global existence result is then derived when the potential function $G$ is bounded from below. The existence of the Lyapounov function $\mathcal{E}$ will be a crucial tool for the analysis of the asymptotic behavior of $(\mathcal{S})$.

Proposition 2.2. Let $a: \mathbb{R}_{+} \rightarrow \mathbb{R}_{+}$be a continuous map and let $G: H \rightarrow \mathbb{R}$ be a function of class $\mathcal{C}^{1}$ such that $\nabla G$ is Lipschitz continuous on the bounded sets of $H$. Let $x$ be a solution to $(\mathcal{S})$ defined on some interval $[0, T)$, with $T \leq \infty$.

(a) For every $t \in[0, T)$, the following equality holds:

$$
\frac{d}{d t} \mathcal{E}(t)=-a(t)|\dot{x}(t)|^{2}
$$

and therefore for $0 \leq s<t<T$,

$$
\mathcal{E}(s)-\int_{s}^{t} a(\tau)|\dot{x}(\tau)|^{2} d \tau=\mathcal{E}(t) .
$$

(b) If in addition $G$ is bounded from below on $H$, then

$$
\int_{0}^{T} a(t)|\dot{x}(t)|^{2} d t<\infty
$$

and the solution exists for all $T>0$.

(c) If also $G$ is coercive 1 then all solutions to $(\mathcal{S})$ remain bounded together with their first and second derivatives for all $t>0$. The bound depends only on the initial data.

Proof. (a) Equation (6) follows by taking the scalar product of $(\mathcal{S})$ against $\dot{x}(t)$, and (7) follows by integrating.

(b) If $G$ is bounded from below, then equality (6) shows that $t \mapsto \mathcal{E}(t)$ is decreasing and remains bounded. Estimate (8) is then a consequence of equality (7), and it also follows that $\sup _{t<T} \mathcal{E}(t)<\infty$. Therefore $\dot{x}$ is uniformly bounded on $[0, T)$. If

\footnotetext{
${ }^{1}$ Let us recall that the coercivity of $G$ means that $G(\xi) \rightarrow \infty$ as $|\xi| \rightarrow \infty$.
} 
$T<\infty$, then the solution $x$ together with its derivative has a limit at $t=T$ and therefore can be continued. Thus the solution $x(t)$ exists for all $t$ and $\dot{x}$ is uniformly bounded by quantities depending on the initial data.

(c) Using the coercivity of $G$ and the inequality $G(x(t)) \leq \mathcal{E}(t) \leq \mathcal{E}(0)$, we derive that the map $x$ is uniformly bounded. Then also $\ddot{x}(t)$ is uniformly bounded due to the differential equation $(\mathcal{S})$ and this bound depends only on the initial data.

If $a$ does not decrease to 0 too rapidly, then the derivative of any solution of $(\mathcal{S})$ must be arbitrarily small on arbitrarily long time intervals, infinitely often.

Proposition 2.3. Let $a: \mathbb{R}_{+} \rightarrow \mathbb{R}_{+}$be a nonincreasing map such that $\int_{0}^{\infty} a(s) d s=$ $\infty$. Let $G: H \rightarrow \mathbb{R}$ be a coercive function of class $\mathcal{C}^{1}$ such that $\nabla G$ is Lipschitz continuous on the bounded sets of $H$. Then, any solution $x$ to the differential equation $(\mathcal{S})$ satisfies, for every $T>0$,

$$
\liminf _{t \rightarrow \infty} \sup _{s \in[t, t+T]}|\dot{x}(s)|=0 .
$$

Proof. Suppose not. Then there exist $\varepsilon>0, T>0$ such that for all $k$,

$$
\sup _{s \in[k T,(k+1) T]}|\dot{x}(s)|>\varepsilon,
$$

and thus there are $t_{k} \in[k T,(k+1) T]$ such that $\left|\dot{x}\left(t_{k}\right)\right|>\varepsilon$. Since the map $\dot{x}$ is Lipschitz continuous, there exists some fixed $\delta>0$ such that $|\dot{x}(t)| \geq \varepsilon / 2$ on $\left[t_{k}-\delta, t_{k}+\delta\right]$ for every $k \in \mathbb{N}$. Since the map $a$ is nonincreasing, we have

$\sum_{k} a((k+1) T) \leq \sum_{k} a\left(t_{k}\right) \leq \sum_{k} \frac{4}{\delta \varepsilon^{2}} \int_{t_{k}-\delta}^{t_{k}} a(t)|\dot{x}(t)|^{2} d t \leq \frac{4}{\delta \varepsilon^{2}} \int_{0}^{\infty} a(t)|\dot{x}(t)|^{2} d t$.

Recalling that $\int_{0}^{\infty} a(t)|\dot{x}(t)|^{2} d t \leq \mathcal{E}(0)-\inf G<\infty$, we infer that $\int_{0}^{\infty} a(t) d t<\infty$, a contradiction.

We next show that if $\dot{x}$ is small on some interval, then $g(x(t))$ is proportionally small on a slightly shorter interval. This implies that if solutions slow down for a long time interval, they must be near a critical point of $G$.

Proposition 2.4. Let $a: \mathbb{R}_{+} \rightarrow \mathbb{R}_{+}$be a nonincreasing map. Let $G: H \rightarrow \mathbb{R}$ be a function of class $\mathcal{C}^{1}$ such that $g=\nabla G$ is Lipschitz continuous on the bounded sets of $H$. If $x$ is a solution of $(\mathcal{S})$ and $|\dot{x}(t)| \leq \varepsilon$ on $\left[T_{0}, T_{1}\right]$, then for every $\left.\left.\delta \in\right] 0, \frac{T_{1}-T_{0}}{2}\right]$, we have

$$
\forall t \in\left[T_{0}+\delta, T_{1}-\delta\right], \quad|g(x(t))| \leq\left(\frac{2}{\delta}+a\left(T_{0}\right)+\frac{L \delta}{2}\right) \varepsilon,
$$

where $L>0$ is a Lipschitz constant of the map $g$ on the set $x\left(\left[T_{0}, T_{1}\right]\right)$.

Proof. Suppose $|\dot{x}(t)| \leq \varepsilon$ on $\left[T_{0}, T_{1}\right]$ with $T_{1}-T_{0} \geq 2 \delta$. Let the map $t \mapsto|g(x(t))|$ attain its maximum on $\left[T_{0}+\delta, T_{1}-\delta\right]$ at $t=t_{0}$. Since the map $g$ is $L$-Lipschitz continuous on the set $x\left(\left[T_{0}, T_{1}\right]\right)$, we have for every $t \in\left[t_{0}-\delta, t_{0}+\delta\right]$,

$$
\left|g\left(x\left(t_{0}\right)\right)-g(x(t))\right| \leq L\left|x\left(t_{0}\right)-x(t)\right| \leq L \varepsilon\left|t_{0}-t\right|
$$

and thus

$$
\left|\ddot{x}(t)+g\left(x\left(t_{0}\right)\right)\right|=\left|a(t) \dot{x}(t)+g(x(t))-g\left(x\left(t_{0}\right)\right)\right| \leq a\left(T_{0}\right) \varepsilon+L \varepsilon\left|t_{0}-t\right|
$$


for the same range of $t$. We then have

$$
\begin{aligned}
\varepsilon \geq|\dot{x}(t)| & \geq\left|\int_{t_{0}}^{t} \ddot{x}(s) d s\right|-\left|\dot{x}\left(t_{0}\right)\right| \\
& \geq\left|\int_{t_{0}}^{t} g\left(x\left(t_{0}\right)\right) d s\right|-\int_{t_{0}}^{t}\left|\ddot{x}(s)+g\left(x\left(t_{0}\right)\right)\right| d s-\varepsilon \\
& \geq\left|t_{0}-t\right|\left|g\left(x\left(t_{0}\right)\right)\right|-\varepsilon-a\left(T_{0}\right) \varepsilon\left|t_{0}-t\right|-\frac{L}{2} \varepsilon\left|t_{0}-t\right|^{2}
\end{aligned}
$$

and therefore

$$
\left|g\left(x\left(t_{0}\right)\right)\right| \leq\left(\frac{2}{\left|t_{0}-t\right|}+a\left(T_{0}\right)+\frac{L}{2}\left|t_{0}-t\right|\right) \varepsilon .
$$

Set $t=t_{0} \pm \delta$ to conclude $\left|g\left(x\left(t_{0}\right)\right)\right| \leq\left(\frac{2}{\delta}+a\left(T_{0}\right)+\frac{L \delta}{2}\right) \varepsilon$.

By combining Propositions 2.3 and 2.4, we derive the following corollary.

Corollary 2.1. Under the assumptions of Proposition 2.3, any solution $x$ to the differential equation $(\mathcal{S})$ satisfies, for every $T>0$,

$$
\liminf _{t \rightarrow \infty} \sup _{s \in[t, t+T]}|g(x(s))|=0 .
$$

The proof is immediate and left to the reader.

We now establish that, if a solution $x$ to $(\mathcal{S})$ converges toward some $\bar{x} \in H$, then $\bar{x}$ is a stationary point of $\nabla G$ and moreover the velocity $\dot{x}(t)$ and the acceleration $\ddot{x}(t)$ tend to 0 as $t \rightarrow \infty$.

Proposition 2.5. Let $a: \mathbb{R}_{+} \rightarrow \mathbb{R}_{+}$be a bounded continuous map and let $G: H \rightarrow$ $\mathbb{R}$ be a function of class $\mathcal{C}^{1}$ such that $\nabla G$ is Lipschitz continuous on the bounded sets of $H$. Consider a solution $x$ to $(\mathcal{S})$ and assume that there exists $\bar{x} \in H$ such that $\lim _{t \rightarrow \infty} x(t)=\bar{x}$. Then we have $\lim _{t \rightarrow \infty} \dot{x}(t)=\lim _{t \rightarrow \infty} \ddot{x}(t)=0$ and the vector $\bar{x}$ satisfies $\nabla G(\bar{x})=0$.

Proof. Since $x(\cdot)$ converges, it is uniformly bounded. Due to the inequality $\mathcal{E}(t) \leq$ $\mathcal{E}(0)$ for every $t \geq 0, \dot{x}(\cdot)$ is also uniformly bounded, and just as in the proof of Proposition 2.2 (c), the map $\ddot{x}(\cdot)$ is uniformly bounded as well by some constant $M>0$. Landau's inequality applied to the map $t \mapsto x(t)-\bar{x}$ yields, for every $t \geq 0$,

$$
\sup _{[t, \infty[}|\dot{x}| \leq 2 \sqrt{\sup _{[t, \infty[}|x-\bar{x}| \cdot \sup _{[t, \infty[}|\ddot{x}|} \leq 2 \sqrt{M} \sqrt{\sup _{[t, \infty[}|x-\bar{x}|} .
$$

By using the assumption $\lim _{t \rightarrow \infty} x(t)=\bar{x}$ and letting $t \rightarrow \infty$ in the above inequality, we derive that $\lim _{t \rightarrow \infty} \dot{x}(t)=0$. Since $\lim _{t \rightarrow \infty} \nabla G(x(t))=\nabla G(\bar{x})$, the differential equation $(\mathcal{S})$ shows that $\lim _{t \rightarrow \infty} \ddot{x}(t)=-\nabla G(\bar{x})$. If $\nabla G(\bar{x}) \neq 0$, an immediate integration gives the equivalence $\dot{x}(t) \sim-t \nabla G(\bar{x})$ as $t \rightarrow \infty$, a contradiction. Thus we conclude that $\nabla G(\bar{x})=0$ and $\lim _{t \rightarrow \infty} \ddot{x}(t)=0$.

The next result provides a lower bound for the convergence rate of the energy function $\mathcal{E}$.

Proposition 2.6. Let $a: \mathbb{R}_{+} \rightarrow \mathbb{R}_{+}$be a continuous map and let $G: H \rightarrow \mathbb{R}$ be a function of class $\mathcal{C}^{1}$ such that $\nabla G$ is Lipschitz continuous on the bounded sets of $H$. If $\inf G>-\infty$, then any solution $x$ of $(\mathcal{S})$ satisfies

$$
\forall t \geq 0, \quad \mathcal{E}(t)-\inf G \geq(\mathcal{E}(0)-\inf G) \cdot e^{-2 \int_{0}^{t} a(s) d s} .
$$


Proof. Taking into account the expression of $\mathcal{E}$ and the computation of $\dot{\mathcal{E}}$, we have

$$
\dot{\mathcal{E}}(t)+2 a(t)(\mathcal{E}(t)-\inf G)=2 a(t)(G(x(t))-\inf G) \geq 0 .
$$

Multiplying the above inequality by $e^{2 \int_{0}^{t} a(s) d s}$, we deduce that

$$
\forall t \geq 0, \quad \frac{d}{d t}\left[e^{2 \int_{0}^{t} a(s) d s}(\mathcal{E}(t)-\min G)\right] \geq 0 .
$$

Formula (9) follows immediately.

By combining Propositions 2.5 and 2.6, we obtain a first result of nonconvergence of the trajectories under the condition $\int_{0}^{\infty} a(s) d s<\infty$. This hypothesis means that the quantity $a(t)$ rapidly tends to 0 as $t \rightarrow \infty$. It is not surprising that convergence fails under such a condition; $c f$. for example the extreme case $a \equiv 0$.

Corollary 2.2. Assume that $\int_{0}^{\infty} a(s) d s<\infty$, that the function $G$ is convex, and assume all the other hypotheses of Proposition 2.6. Given $\left(x_{0}, \dot{x}_{0}\right) \in H^{2}$, consider the unique solution $x$ to the differential equation $(\mathcal{S})$ satisfying the initial conditions $(x(0), \dot{x}(0))=\left(x_{0}, \dot{x}_{0}\right)$. If $\left(x_{0}, \dot{x}_{0}\right) \notin \arg \min G \times\{0\}$, then the trajectory $x$ of $(\mathcal{S})$ does not converge.

Proof. Let us first remark that the assumption $\left(x_{0}, \dot{x}_{0}\right) \notin \arg \min G \times\{0\}$ implies that $\mathcal{E}(0)>\inf G$. By taking the limit as $t \rightarrow \infty$ in inequality (9) and recalling that $\int_{0}^{\infty} a(s) d s<\infty$, we obtain

$$
\lim _{t \rightarrow \infty} \mathcal{E}(t)-\inf G \geq(\mathcal{E}(0)-\inf G) \cdot e^{-2 \int_{0}^{\infty} a(s) d s}>0 .
$$

Let us now argue by contradiction and assume that there exists $\bar{x} \in H$ such that $\lim _{t \rightarrow \infty} x(t)=\bar{x}$. From Proposition 2.5 we deduce that $\lim _{t \rightarrow \infty} \dot{x}(t)=0$ and that $\nabla G(\bar{x})=0$. Since the function $G$ is convex, we infer that $\bar{x} \in \arg \min G$. It ensues that $\lim _{t \rightarrow \infty} \mathcal{E}(t)=\min G$, which contradicts (10).

It will be shown in section 4 that condition $\int_{0}^{\infty} a(s) d s=\infty$ is not sufficient to ensure convergence.

\section{The case of a convex-like potential. Energy estimates}

As in the previous section, $(\mathcal{S})$ is studied on a general Hilbert space $H$. Throughout, we will assume that the function $G$ satisfies the following condition: there exist $z \in \arg \min G$ and $\theta \in \mathbb{R}_{+}$such that

$$
\forall x \in H, \quad G(x)-G(z) \leq \theta\langle\nabla G(x), x-z\rangle .
$$

This can be viewed as a generalization of the notion of convexity, and it also generalizes Euler's identity for homogeneous functions. Indeed, if $G$ is convex, then condition (11) is satisfied with $\theta=1$ for every $z \in H$. Now assume that $G$ is defined by $G(x)=\frac{1}{2}\langle A x, x\rangle$, where, $A \in \mathcal{L}(H)$ is symmetric and positive. We then have $g(x)=A x$ and inequality (11) holds as an equality with $\theta=\frac{1}{2}$ and $z=0$. Finally, if $G$ is defined by $G(x)=\frac{|x|^{p}}{p}$ with $p>1$, we have $g(x)=x|x|^{p-2}$ and inequality (11) is satisfied with $\theta=\frac{1}{p}$ and $z=0$ as an equality. 
3.1. A result of summability. First we give a result of summability for the function $t \mapsto a(t)(\mathcal{E}(t)-\min G)$ over $\mathbb{R}$. This property will imply some convergence results on $\mathcal{E}$ provided some weak hypotheses hold for the function $a$.

Proposition 3.1. Let $a: \mathbb{R}_{+} \rightarrow \mathbb{R}_{+}$be a nonincreasing and differentiable map. Let $G: H \rightarrow \mathbb{R}$ be a coercive function of class $\mathcal{C}^{1}$ such that $\nabla G$ is Lipschitz continuous on the bounded sets of $H$. Assume that $\arg \min G \neq \emptyset$ and that there exist $z \in$ $\arg \min G$ and $\theta \in \mathbb{R}_{+}$such that condition (11) holds. Then, any solution $x$ to the differential equation $(\mathcal{S})$ satisfies the following estimate:

$$
\int_{0}^{\infty} a(t)(\mathcal{E}(t)-\min G) d t<\infty
$$

Proof. Let us define the function $h: \mathbb{R}_{+} \rightarrow \mathbb{R}$ by

$$
h(t)=\frac{a(t)}{2}|x(t)-z|^{2}+\langle\dot{x}(t), x(t)-z\rangle .
$$

By differentiating, we find:

$$
\dot{h}(t)=\frac{\dot{a}(t)}{2}|x(t)-z|^{2}+a(t)\langle\dot{x}(t), x(t)-z\rangle+\langle\ddot{x}(t), x(t)-z\rangle+|\dot{x}(t)|^{2} .
$$

Since $\dot{a}(t) \leq 0$, we derive that

$$
\begin{aligned}
\dot{h}(t) & \leq|\dot{x}(t)|^{2}+\langle\ddot{x}(t)+a(t) \dot{x}(t), x(t)-z\rangle \\
& \leq|\dot{x}(t)|^{2}-\langle g(x(t)), x(t)-z\rangle .
\end{aligned}
$$

Let us now fix $\left.m \in] 0, \frac{1}{\theta+1 / 2}\right]$. Recalling that $\dot{\mathcal{E}}(t)=-a(t)|\dot{x}(t)|^{2}$, we find

$$
\begin{aligned}
& \dot{\mathcal{E}}(t)+m a(t)(\mathcal{E}(t)-\min G)+\theta m a(t) \dot{h}(t) \\
& =(-1+(\theta+1 / 2) m) a(t)|\dot{x}(t)|^{2}+m a(t)[G(x(t))-\min G-\theta\langle g(x(t)), x(t)-z\rangle] .
\end{aligned}
$$

Using condition (11) and the fact that $m \leq \frac{1}{\theta+1 / 2}$, we deduce

$$
\dot{\mathcal{E}}(t)+m a(t)(\mathcal{E}(t)-\min G)+\theta m a(t) \dot{h}(t) \leq 0 .
$$

Let us integrate the previous inequality on $[0, t]$. Since $\mathcal{E}(t) \geq \min G$, we obtain

$$
m \int_{0}^{t} a(s)(\mathcal{E}(s)-\min G) d s \leq \mathcal{E}(0)-\min G-\theta m \int_{0}^{t} a(s) \dot{h}(s) d s .
$$

Then, remark that

$$
\int_{0}^{t} a(s) \dot{h}(s) d s=a(t) h(t)-a(0) h(0)-\int_{0}^{t} \dot{a}(s) h(s) d s .
$$

From the decay of the energy function $\mathcal{E}$, it ensues that $t \mapsto|\dot{x}(t)|$ and $t \mapsto G(x(t))$ are bounded. Since the map $G$ is coercive, we infer that the map $t \mapsto|x(t)|$ is bounded. From the expression of $h$, and the boundedness of $t \mapsto \mathcal{E}(t)$ and thus of $t \mapsto|\dot{x}(t)|$, we immediately conclude the existence of $M>0$ such that $|h(t)| \leq M$ for every $t \geq 0$. We then derive from (14) that

$$
\begin{aligned}
\left|\int_{0}^{t} a(s) \dot{h}(s) d s\right| & \leq M a(t)+M a(0)+M \int_{0}^{t}|\dot{a}(s)| d s \\
& =M a(t)+M a(0)+M(a(0)-a(t))=2 M a(0) .
\end{aligned}
$$


From (13), we now have that

$$
\forall t \geq 0, \quad m \int_{0}^{t} a(s)(\mathcal{E}(s)-\min G) d s \leq \mathcal{E}(0)-\min G+2 \theta m M a(0)
$$

and we conclude that $\int_{0}^{\infty} a(s)(\mathcal{E}(s)-\min G) d s<\infty$.

Now, we can prove the convergence of $\mathcal{E}(t)$ toward $\min G$ as $t \rightarrow \infty$, provided that $\int_{0}^{\infty} a(t) d t=\infty$. Notice that this assumption amounts to saying that the quantity $a(t)$ slowly tends to 0 as $t \rightarrow \infty$.

Corollary 3.1. Under the hypotheses of Proposition 3.1, assume moreover that $\int_{0}^{\infty} a(t) d t=\infty$. Then $\lim _{t \rightarrow \infty} \mathcal{E}(t)=\min G$. As a consequence, $\lim _{t \rightarrow \infty}|\dot{x}(t)|=0$ and $\lim _{t \rightarrow \infty} G(x(t))=\min G$.

Proof. Let us argue by contradiction and assume that $\lim _{t \rightarrow \infty} \mathcal{E}(t)>\min G$. This implies the existence of $\eta>0$ such that $\mathcal{E}(t)-\min G \geq \eta$ for every $t \geq 0$. We deduce that

$$
\int_{0}^{\infty} a(t)(\mathcal{E}(t)-\min G) d t \geq \eta \int_{0}^{\infty} a(t) d t=\infty .
$$

This yields a contradiction and we obtain the conclusions that $\lim _{t \rightarrow \infty}|\dot{x}(t)|=0$ and $\lim _{t \rightarrow \infty} G(x(t))=\min G$.

The next corollary specifies the speed of convergence of $\mathcal{E}$ toward $\min G$ under some assumption on the decay of $t \mapsto a(t)$.

Corollary 3.2. Under the hypotheses of Proposition 3.1, assume moreover that there exists $m>0$ such that $a(t) \geq m / t$ for $t$ large enough. Then

$$
\mathcal{E}(t)-\min G=o\left(\frac{1}{t a(t)}\right) \quad \text { as } t \rightarrow \infty .
$$

Proof. Since the functions $a$ and $\mathcal{E}$ are nonincreasing and positive, it is immediate that the map $t \mapsto a(t)(\mathcal{E}(t)-\min G)$ is also nonincreasing. In particular, we obtain

$$
\int_{t / 2}^{t} a(s)(\mathcal{E}(s)-\min G) d s \geq \frac{t}{2} a(t)(\mathcal{E}(t)-\min G) .
$$

Since $\int_{0}^{\infty} a(s)(\mathcal{E}(s)-\min G) d s<\infty$, the left member of the above inequality tends to 0 as $t \rightarrow \infty$, which implies that $\lim _{t \rightarrow \infty} \operatorname{ta}(t)(\mathcal{E}(t)-\min G)=0$.

3.2. Case of a unique minimum. In view of the previous results, we are able to investigate the question of the convergence of the trajectories in the case of a unique minimum. Studies with several minima will be detailed in section 4 (convex setting), section 5 (nonconvex setting) and section 6 (one-dimensional case).

Proposition 3.2. Let $a: \mathbb{R}_{+} \rightarrow \mathbb{R}_{+}$be a nonincreasing and differentiable map such that $\int_{0}^{\infty} a(t) d t=\infty$. Consider a map $\alpha: \mathbb{R}_{+} \rightarrow \mathbb{R}_{+}$such that $\alpha\left(t_{n}\right) \rightarrow 0$ $\Longrightarrow t_{n} \rightarrow 0$ for every sequence $\left(t_{n}\right) \subset \mathbb{R}_{+}$. Let $G: H \rightarrow \mathbb{R}$ be a coercive function of class $\mathcal{C}^{1}$ such that $\nabla G$ is Lipschitz continuous on the bounded sets of $H$. Given $\bar{x} \in H$, assume that

$$
\forall x \in H, \quad G(x) \geq G(\bar{x})+\alpha(|x-\bar{x}|),
$$

and that there exists $\theta \in \mathbb{R}_{+}$such that condition (11) holds with $z=\bar{x}$. Then, any solution $x$ to the differential equation $(\mathcal{S})$ satisfies $\lim _{t \rightarrow \infty} x(t)=\bar{x}$ strongly in $H$. 
Proof. By applying Corollary [3.1. we obtain $\lim _{t \rightarrow \infty} G(x(t))=\min G=G(\bar{x})$. From assumption (15), we deduce that $\lim _{t \rightarrow \infty} \alpha(|x(t)-\bar{x}|)=0$ and we finally conclude that $\lim _{t \rightarrow \infty}|x(t)-\bar{x}|=0$.

If the stringent condition (15) is not satisfied, one can nevertheless obtain a result of weak convergence, as shown by the following statement.

Proposition 3.3. Let $a: \mathbb{R}_{+} \rightarrow \mathbb{R}_{+}$be a nonincreasing and differentiable map such that $\int_{0}^{\infty} a(t) d t=\infty$. Let $G: H \rightarrow \mathbb{R}$ be a convex coercive function of class $\mathcal{C}^{1}$ such that $\nabla G$ is Lipschitz continuous on the bounded sets of $H$. If $\arg \min G=\{\bar{x}\}$ for some $\bar{x} \in H$, then any solution $x$ to the differential equation $(\mathcal{S})$ weakly converges to $\bar{x}$ in $H$.

Proof. Since $G$ is coercive, the trajectory $x$ is bounded. Hence there exist $x_{\infty} \in H$ and a subsequence $\left(t_{n}\right)$ tending to $\infty$ such that $\lim _{n \rightarrow \infty} x\left(t_{n}\right)=x_{\infty}$ weakly in $H$. Since $G$ is convex and continuous for the strong topology, it is lower semicontinuous for the weak topology. Hence, we have

$$
G\left(x_{\infty}\right) \leq \liminf _{n \rightarrow \infty} G\left(x\left(t_{n}\right)\right) .
$$

On the other hand, by applying Corollary 3.1 we obtain $\lim _{t \rightarrow \infty} G(x(t))=\min G$. Therefore we deduce that $G\left(x_{\infty}\right) \leq \min G$, i.e. $x_{\infty} \in \arg \min G=\{\bar{x}\}$. Hence $\bar{x}$ is the unique limit point of the map $t \mapsto x(t)$ as $t \rightarrow \infty$ for the weak topology. It ensues that $\lim _{t \rightarrow \infty} x(t)=\bar{x}$ weakly in $H$.

3.3. Convergence rate of the energy function $\mathcal{E}$. Let us start with the particular case corresponding to $G(x)=|x|^{2} / 2$. Equation $(\mathcal{S})$ then becomes

$$
\ddot{x}(t)+a(t) \dot{x}(t)+x(t)=0 .
$$

The next proposition makes precise the rate at which solutions converge to 0 .

Proposition 3.4. Let $a: \mathbb{R}_{+} \rightarrow \mathbb{R}_{+}$be a nonincreasing map of class $\mathcal{C}^{2}$. Assume that $\lim _{t \rightarrow \infty} a(t)=\lim _{t \rightarrow \infty} \dot{a}(t)=0$ and that the map $t \mapsto \ddot{a}(t)+a(t) \dot{a}(t)$ has a constant sign when $t \rightarrow \infty$. Let $x$ be a solution of the differential equation (16). Then there exist constants $0<k<K<\infty$ such that for $t$ large enough,

$$
k e^{-\int_{0}^{t} a(s) d s} \leq|x(t)|^{2}+|\dot{x}(t)|^{2} \leq K e^{-\int_{0}^{t} a(s) d s} .
$$

Proof. We eliminate the first order term in (16) in the usual way: if we set $A(t)=$ $e^{\frac{1}{2} \int_{0}^{t} a(s) d s}$, then the map $y$ defined by $y(t)=A(t) x(t)$ satisfies

$$
\ddot{y}(t)+\left(1-\frac{\dot{a}(t)}{2}-\frac{a(t)^{2}}{4}\right) y(t)=0
$$

for every $t \geq 0$. Define the function $E: \mathbb{R}_{+} \rightarrow \mathbb{R}$ by

$$
E(t)=|\dot{y}(t)|^{2}+\left(1-\frac{\dot{a}(t)}{2}-\frac{a(t)^{2}}{4}\right)|y(t)|^{2}
$$

for every $t \geq 0$. Then $E(t)$ is nonnegative for all sufficiently large $t$, and the expression of $E(t)$ as a function of $x(t), \dot{x}(t)$ is given by

$$
E(t)=A(t)^{2}\left(\left(1-\frac{\dot{a}(t)}{2}-\frac{a(t)^{2}}{4}\right)|x(t)|^{2}+\left|\frac{a(t)}{2} x(t)+\dot{x}(t)\right|^{2}\right) .
$$


Therefore for sufficiently large $t$,

$$
\frac{A(t)^{-2}}{2} E(t) \leq|x(t)|^{2}+|\dot{x}(t)|^{2} \leq 2 A(t)^{-2} E(t) .
$$

Multiplying equation (18) with $\dot{y}(t)$ results in

$$
\dot{E}(t)=-\frac{1}{2}[\ddot{a}(t)+a(t) \dot{a}(t)]|y(t)|^{2} .
$$

Assume now that $\ddot{a}(t)+a(t) \dot{a}(t) \leq 0$ for $t$ large enough. Since $|y(t)|^{2} \leq 2 E(t)$ for sufficiently large $t$, we derive that there exists $T \geq 0$ such that

$$
\forall t \geq T, \quad 0 \leq \dot{E}(t) \leq-[\ddot{a}(t)+a(t) \dot{a}(t)] E(t) .
$$

By integrating over $[T, t]$, we obtain

$$
\forall t \geq T, \quad 0 \leq \ln \frac{E(t)}{E(T)} \leq-\left[\dot{a}(s)+\frac{a^{2}(s)}{2}\right]_{T}^{t} \leq \dot{a}(T)+\frac{a^{2}(T)}{2} .
$$

By setting $C=\exp \left(\dot{a}(T)+\frac{a^{2}(T)}{2}\right)$, we then have

$$
\forall t \geq T, \quad E(T) \leq E(t) \leq C E(T) .
$$

Then estimate (17) follows from (19) and (20). If we assume that $\ddot{a}(t)+a(t) \dot{a}(t) \geq 0$ for $t$ large enough, the same arguments show that there exist $T^{\prime} \geq 0$ and $C^{\prime} \in(0,1)$ such that

$$
\forall t \geq T^{\prime}, \quad C^{\prime} E(T) \leq E(t) \leq E(T),
$$

and we conclude the proof in the same way.

Example 3.1. Assume that $a(t)=\frac{c}{t+1}$ for every $t \geq 0$, with $c>0$. It is immediate to check that for $t$ large enough, $\ddot{a}(t)+a(t) \dot{a}(t) \geq 0$ (resp. $\leq 0$ ) if $c \leq 2$ (resp. $c \geq 2$ ). Therefore the assumptions of Proposition 3.4 are satisfied and the following estimate holds for $t$ large enough:

$$
\frac{k}{t^{c}} \leq|x(t)|^{2}+|\dot{x}(t)|^{2} \leq \frac{K}{t^{c}}
$$

Example 3.2. Assume that $a(t)=\frac{1}{(t+1)^{\alpha}}$ for every $t \geq 0$, with $\alpha \in(0,1)$. We let the reader check that $\ddot{a}(t)+a(t) \dot{a}(t) \leq 0$ for $t$ large enough. Therefore the assumptions of Proposition 3.4 are satisfied and the following estimate holds for $t$ large enough:

$$
k e^{-t^{1-\alpha} /(1-\alpha)} \leq|x(t)|^{2}+|\dot{x}(t)|^{2} \leq K e^{-t^{1-\alpha} /(1-\alpha)} .
$$

Let us now come back to the case of a general potential $G$. We are going to find upper bounds for the speed of convergence of the map $t \mapsto \mathcal{E}(t)-\inf G$ as $t \rightarrow \infty$.

Proposition 3.5. Let $a: \mathbb{R}_{+} \rightarrow \mathbb{R}_{+}$be a nonincreasing and differentiable map. Let $G: H \rightarrow \mathbb{R}$ be a coercive function of class $\mathcal{C}^{1}$ such that $\nabla G$ is Lipschitz continuous on the bounded sets of $H$. Assume that $\arg \min G \neq \emptyset$ and that there exist $z \in$ $\arg \min G$ and $\theta \in \mathbb{R}_{+}$such that condition (11) holds.

(i) Suppose that there exist $K_{1}>0$ and $t_{1} \geq 0$ such that $\dot{a}(t)+K_{1} a^{2}(t) \leq 0$, for every $t \geq t_{1}$. Then, there exists $C>0$ such that, for every $t \geq t_{1}$,

$$
\mathcal{E}(t)-\min G \leq C e^{-m \int_{0}^{t} a(s) d s},
$$

with $m=\min \left(\frac{1}{\theta+1 / 2}, K_{1}\right)$. 
(ii) Suppose that there exist $\left.\left.K_{2} \in\right] 0, \frac{1}{\theta+1 / 2}\right]$ and $t_{2} \geq 0$ such that $\dot{a}(t)+$ $K_{2} a^{2}(t) \geq 0$, for every $t \geq t_{2}$. Then, there exists $D>0$ such that, for every $t \geq t_{2}$

$$
\mathcal{E}(t)-\min G \leq D a(t) .
$$

Remark 3.1. It is immediate to see that the assumptions $\dot{a}+K_{1} a^{2} \leq 0$ and $\dot{a}+$ $K_{2} a^{2} \geq 0$ imply respectively that $a(t) \leq 1 /\left(K_{1} t+c_{1}\right)$ and $a(t) \geq 1 /\left(K_{2} t+c_{2}\right)$, for some $c_{1}, c_{2} \in \mathbb{R}$.

Proof. We keep the same notation as in the proof of Proposition 3.1 in particular, the expression of the map $h$ is given by

$$
h(t)=\frac{a(t)}{2}|x(t)-z|^{2}+\langle\dot{x}(t), x(t)-z\rangle .
$$

Let us multiply inequality (12) by $e^{m \int_{0}^{t} a(s) d s}$ and integrate on the interval $[0, t]$ :

$$
e^{m \int_{0}^{t} a(s) d s}(\mathcal{E}(t)-\min G) \leq \mathcal{E}(0)-\min G-\theta m \int_{0}^{t} F(s) \dot{h}(s) d s,
$$

where the function $F: \mathbb{R}_{+} \rightarrow \mathbb{R}_{+}$is defined by $F(s)=a(s) e^{m \int_{0}^{s} a(u) d u}$. The function $F$ is differentiable and its first derivative is given by

$$
\dot{F}(s)=\left(\dot{a}(s)+m a^{2}(s)\right) e^{m \int_{0}^{s} a(u) d u} .
$$

Coming back to inequality (23), an integration by parts yields

$$
\int_{0}^{t} F(s) \dot{h}(s) d s=F(t) h(t)-F(0) h(0)-\int_{0}^{t} \dot{F}(s) h(s) d s .
$$

Recalling that $|h(t)| \leq M$ for every $t \geq 0$, we infer that

$$
\left|\int_{0}^{t} F(s) \dot{h}(s) d s\right| \leq M\left[F(t)+F(0)+\int_{0}^{t}|\dot{F}(s)| d s\right] .
$$

We now distinguish between the cases (i) and (ii), where the assumptions allow us to determine the sign of $\dot{F}$.

(i) First assume that there exist $K_{1}>0$ and $t_{1} \geq 0$ such that $\dot{a}(t)+K_{1} a^{2}(t) \leq 0$, for every $t \geq t_{1}$. Let us take $m=\min \left(\frac{1}{\theta+1 / 2}, K_{1}\right)$ throughout the proof of (i). Since $m \leq K_{1}$, we have $\dot{a}(t)+m a^{2}(t) \leq \dot{a}(t)+K_{1} a^{2}(t) \leq 0$ for every $t \geq t_{1}$. It ensues from (24) that $\dot{F}(t) \leq 0$ for every $t \geq t_{1}$. Hence we derive from (25) that, for every $t \geq t_{1}$,

$$
\begin{aligned}
\left|\int_{0}^{t} F(s) \dot{h}(s) d s\right| & \leq M\left[F(t)+F(0)+\int_{0}^{t_{1}}|\dot{F}(s)| d s+\int_{t_{1}}^{t}|\dot{F}(s)| d s\right] \\
& =M\left[F(0)+\int_{0}^{t_{1}}|\dot{F}(s)| d s+F\left(t_{1}\right)\right]=C_{1} .
\end{aligned}
$$

In view of (23), we deduce that, for every $t \geq t_{1}$,

$$
e^{m \int_{0}^{t} a(s) d s}(\mathcal{E}(t)-\min G) \leq \mathcal{E}(0)-\min G+\theta m C_{1}=C .
$$

Inequality (21) immediately follows.

(ii) Now assume that there exist $\left.\left.K_{2} \in\right] 0, \frac{1}{\theta+1 / 2}\right]$ and $t_{2} \geq 0$ such that $\dot{a}(t)+$ $K_{2} a^{2}(t) \geq 0$, for every $t \geq t_{2}$. Take any $m \in\left[K_{2}, \frac{1}{\theta+1 / 2}\right]$. Since $m \geq K_{2}$, we 
have $\dot{a}(t)+m a^{2}(t) \geq \dot{a}(t)+K_{2} a^{2}(t) \geq 0$ for every $t \geq t_{2}$. It ensues from (24) that $\dot{F}(t) \geq 0$ for every $t \geq t_{2}$. Hence we derive from (25) that, for every $t \geq t_{2}$,

$$
\begin{aligned}
\left|\int_{0}^{t} F(s) \dot{h}(s) d s\right| & \leq M\left[F(t)+F(0)+\int_{0}^{t_{2}}|\dot{F}(s)| d s+\int_{t_{2}}^{t}|\dot{F}(s)| d s\right] \\
& =M\left[2 F(t)+F(0)-F\left(t_{2}\right)+\int_{0}^{t_{2}}|\dot{F}(s)| d s\right]=2 M F(t)+C_{2} .
\end{aligned}
$$

In view of (23), we deduce that, for every $t \geq t_{2}$,

$$
e^{m \int_{0}^{t} a(s) d s}(\mathcal{E}(t)-\min G) \leq \mathcal{E}(0)-\min G+\theta m C_{2}+2 \theta m M F(t) .
$$

We then infer the existence of $C_{3}>0$ such that

$$
e^{m \int_{0}^{t} a(s) d s}(\mathcal{E}(t)-\min G) \leq C_{3} F(t),
$$

which finally implies that $\mathcal{E}(t)-\min G \leq C_{3} a(t)$, for every $t \geq t_{2}$.

Let us now comment on the results given by Proposition 3.5. Assume that $G$ is identically equal to 0 . In this case, condition (11) trivially holds with $\theta=0$. If the map $a$ satisfies $\dot{a}+K_{1} a^{2} \leq 0$ for some $K_{1} \geq 2$, Proposition 3.5 (i) shows that

$$
\frac{1}{2}|\dot{x}(t)|^{2}=\mathcal{E}(t) \leq C e^{-2 \int_{0}^{t} a(s) d s} .
$$

A direct computation shows that $\dot{x}(t)=\dot{x}_{0} e^{-\int_{0}^{t} a(s) d s}$, so that the estimate given by Proposition 3.5 (i) is optimal in this case. Now assume that $G$ is given by $G(x)=|x|^{2} / 2$ for every $x \in H$. In this case, condition (11) holds with $\theta=1 / 2$ and $z=0$. Suppose that $a(t)=\frac{c}{t+1}$ for $c \in(0,1]$. The map $a$ satisfies the inequality $\dot{a}+K_{1} a^{2} \leq 0$ for every $K_{1} \in\left(0, \frac{1}{c}\right.$ ]. Proposition 3.5 (i) then shows that $\mathcal{E}(t) \leq C /(t+1)^{c}$ for every $t \geq 0$. Example 3.1 allows us to check that this estimate is optimal. Suppose now that $a(t)=\frac{1}{(t+1)^{\alpha}}$ for some $\alpha \in(0,1)$. In this case, the map $a$ satisfies the inequality $\dot{a}+K_{2} a^{2} \geq 0$ for any $K_{2}>0$. Proposition 3.5 (ii) then gives the estimate $\mathcal{E}(t) \leq \frac{D}{(t+1)^{\alpha}}$ while in fact $\mathcal{E}(t) \leq K e^{-t^{1-\alpha} /(1-\alpha)}$ by the result of Example 3.2

\section{The PRoblem of CONVERGENCE OF TRAJECTORIES FOR CONVEX POTENTIALS}

We are going to investigate the question of convergence of the trajectories associated to $(\mathcal{S})$ when the potential $G$ is convex and has nonisolated minima. A first result in this direction is given by Corollary 2.2, which states that the trajectories of $(\mathcal{S})$ are not convergent under the condition $\int_{0}^{\infty} a(s) d s<\infty$ (except for stationary solutions). Let us now consider the particular case $G \equiv 0$. The differential equation $(\mathcal{S})$ then becomes $\ddot{x}(t)+a(t) \dot{x}(t)=0$ and a double integration immediately shows that its solution is given by

$$
x(t)=x(0)+\dot{x}(0) \int_{0}^{t} e^{-\int_{0}^{s} a(u) d u} d s .
$$

It ensues that, when $G \equiv 0$, the solution $x$ converges if and only if the quantity $\int_{0}^{\infty} e^{-\int_{0}^{s} a(u) d u} d s$ is finite. Therefore it is natural to ask whether for a general potential $G$, the trajectory $x$ is convergent under the condition $\int_{0}^{\infty} e^{-\int_{0}^{s} a(u) d u} d s<\infty$. The answer is quite complex, and we will start our analysis in the one-dimensional case. 
4.1. One-dimensional case. First, we give a general result of nonconvergence of the trajectories under the condition

$$
\int_{0}^{\infty} e^{-\int_{0}^{t} a(s) d s} d t=\infty .
$$

Note that it is automatically satisfied if $\int_{0}^{\infty} a(s) d s<\infty$. Condition (26) expresses that the parametrization $a$ tends to zero rather rapidly. For example, assume that the map $a$ is of the form $a(t)=c /(t+1)^{\gamma}$, with $\gamma, c \geq 0$. It is immediate to check that condition (26) is satisfied if and only if $(\gamma, c) \in(1, \infty) \times \mathbb{R}_{+}$or $(\gamma, c) \in\{1\} \times[0,1]$. Let us now state a preliminary result.

Lemma 4.1. Let $a: \mathbb{R}_{+} \rightarrow \mathbb{R}_{+}$be a continuous map such that $\int_{0}^{\infty} e^{-\int_{0}^{t} a(s) d s} d t=\infty$.

(i) Suppose that the map $p \in \mathcal{C}^{2}\left(\left[t_{0}, \infty\right), \mathbb{R}\right)$ satisfies

$$
\forall t \geq t_{0}, \quad \ddot{p}(t)+a(t) \dot{p}(t) \leq 0 .
$$

Then, we have either $\lim _{t \rightarrow \infty} p(t)=-\infty$ or $\dot{p}(t) \geq 0$ for every $t \in\left[t_{0}, \infty[\right.$.

(ii) Assume that the map $p$ satisfies moreover:

$$
\forall t \geq t_{0}, \quad \ddot{p}(t)+a(t) \dot{p}(t)=0 .
$$

Then, either $\lim _{t \rightarrow \infty}|p(t)|=\infty$ or $p(t)=p\left(t_{0}\right)$ for every $t \in\left[t_{0}, \infty[\right.$.

Proof. (i) Assume that there exists $t_{1} \in\left[t_{0}, \infty\left[\right.\right.$ such that $\dot{p}\left(t_{1}\right)<0$. Let us multiply inequality (27) by $e^{\int_{t_{0}}^{t} a(s) d s}$ to obtain

$$
\frac{d}{d t}\left[e^{\int_{t_{0}}^{t} a(s) d s} \dot{p}(t)\right] \leq 0 .
$$

Let us integrate the above inequality on the interval $\left[t_{1}, t\right]$, for $t \geq t_{1}$ :

$$
\dot{p}(t) \leq \dot{p}\left(t_{1}\right) e^{-\int_{t_{1}}^{t} a(s) d s} .
$$

By integrating again, we find

$$
\forall t \geq t_{1}, \quad p(t) \leq p\left(t_{1}\right)+\dot{p}\left(t_{1}\right) \int_{t_{1}}^{t} e^{-\int_{t_{1}}^{s} a(u) d u} d s .
$$

Recalling that $\dot{p}\left(t_{1}\right)<0$ and that $\int_{0}^{\infty} e^{-\int_{0}^{s} a(u) d u} d s=\infty$, we conclude that $\lim _{t \rightarrow \infty} p(t)=-\infty$.

(ii) Assume now that the map $p$ satisfies equality (28) and that there exists $t_{1} \in\left[t_{0}, \infty\left[\right.\right.$ such that $\dot{p}\left(t_{1}\right) \neq 0$. The same computation as above shows that

$$
\forall t \geq t_{1}, \quad p(t)=p\left(t_{1}\right)+\dot{p}\left(t_{1}\right) \int_{t_{1}}^{t} e^{-\int_{t_{1}}^{s} a(u) d u} d s .
$$

Since $\dot{p}\left(t_{1}\right) \neq 0$ and the integral $\int_{0}^{\infty} e^{-\int_{0}^{s} a(u) d u} d s$ is divergent, we conclude that $\lim _{t \rightarrow \infty} p(t)= \pm \infty$ (depending on the sign of $\left.\dot{p}\left(t_{1}\right)\right)$.

Lemma 4.1 is a crucial tool in the proof of the following nonconvergence result.

Proposition 4.1. Let $G: \mathbb{R} \rightarrow \mathbb{R}$ be a convex function of class $\mathcal{C}^{1}$ such that $G^{\prime}$ is Lipschitz continuous on the bounded sets of $\mathbb{R}$. Assume that $\arg \min G=[\alpha, \beta]$, for some $\alpha, \beta \in \mathbb{R}$ such that $\alpha<\beta$. Let $a: \mathbb{R}_{+} \rightarrow \mathbb{R}_{+}$be a continuous map such that $\int_{0}^{\infty} e^{-\int_{0}^{t} a(s) d s} d t=\infty$. Given $\left(x_{0}, \dot{x}_{0}\right) \in \mathbb{R}^{2}$, consider the unique solution $x$ to the differential equation $(\mathcal{S})$ satisfying the initial conditions $(x(0), \dot{x}(0))=\left(x_{0}, \dot{x}_{0}\right)$. If $\left(x_{0}, \dot{x}_{0}\right) \notin[\alpha, \beta] \times\{0\}$, the $\omega$ - limit set $\omega\left(x_{0}, \dot{x}_{0}\right)$ contains $[\alpha, \beta]$; hence the trajectory $x$ does not converge. 
Proof. Let us assume that

$$
\exists t_{0} \geq 0, \quad \forall t \geq t_{0}, \quad x(t) \geq \alpha
$$

and let us prove that this leads to a contradiction. First of all, assertion (29) implies that $G^{\prime}(x(t)) \geq 0$ for every $t \geq t_{0}$, which in view of $(\mathcal{S})$ entails that

$$
\forall t \geq t_{0}, \quad \ddot{x}(t)+a(t) \dot{x}(t) \leq 0 .
$$

Since the map $x$ is bounded, we deduce from Lemma 4.1(i) that $\dot{x}(t) \geq 0$ for every $t \geq t_{0}$; hence $\bar{x}:=\lim _{t \rightarrow \infty} x(t)$ exists. From Proposition 2.5. we have $G^{\prime}(\bar{x})=0$, i.e. $\bar{x} \in[\alpha, \beta]$. It ensues that $x(t) \in[\alpha, \beta]$ for every $t \geq t_{0}$. Hence we have $G^{\prime}(x(t))=0$ for every $t \geq t_{0}$ and we infer that

$$
\forall t \geq t_{0}, \quad \ddot{x}(t)+a(t) \dot{x}(t)=0 .
$$

Since $x\left(\left[t_{0}, \infty[) \subset[\alpha, \beta]\right.\right.$, we derive from Lemma 4.1(ii) that $x(t)=x\left(t_{0}\right)$ for every $t \geq t_{0}$. Thus, it follows by backward uniqueness that we have a stationary solution, which contradicts the assumption $\left(x_{0}, \dot{x}_{0}\right) \notin[\alpha, \beta] \times\{0\}$. Hence, we deduce that assertion (29) is false, so that we can build a sequence $\left(t_{n}\right)$ tending to $\infty$ such that $x\left(t_{n}\right)<\alpha$. In a symmetric way, we can construct a sequence $\left(u_{n}\right)$ tending to $\infty$ such that $x\left(u_{n}\right)>\beta$. Recalling that the $\omega$-limit set $\omega\left(x_{0}, \dot{x}_{0}\right)$ is connected, we conclude that $\omega\left(x_{0}, \dot{x}_{0}\right) \supset[\alpha, \beta]$.

We can now wonder if the converse assertion is true: do the trajectories $x$ of $(\mathcal{S})$ converge under the condition $\int_{0}^{\infty} e^{-\int_{0}^{s} a(u) d u} d s<\infty$ ? When the coefficient $a(t)$ is constant and positive, the trajectories of the so-called "Heavy Ball with Friction" system are known to be convergent; see for example [5. The question is more delicate in the case of an asymptotically vanishing map $a$. We mention below a first positive result when the map $a$ is of the form $a(t)=\frac{c}{(t+1)^{\gamma}}$ with $c, \gamma>0$.

Proposition 4.2. Let $G: \mathbb{R} \rightarrow \mathbb{R}$ be a convex function of class $\mathcal{C}^{1}$ such that $G^{\prime}$ is Lipschitz continuous on the bounded sets of $\mathbb{R}$. Assume that $\arg \min G=[\alpha, \beta]$ with $\alpha \leq \beta$ and that there exists $\delta>0$ such that

$$
\forall \xi \in(-\infty, \alpha], \quad G^{\prime}(\xi) \leq \delta(\xi-\alpha) \quad \text { and } \quad \forall \xi \in[\beta, \infty), \quad G^{\prime}(\xi) \geq \delta(\xi-\beta) .
$$

Given $c, \gamma>0$, let $a: \mathbb{R}_{+} \rightarrow \mathbb{R}_{+}$be the map defined by $a(t)=\frac{c}{(t+1)^{\gamma}}$ for every $t \geq 0$. If $\gamma \in(0,1)$ or if $\gamma=1$ and $c>1$, then for any solution $x$ of $(\mathcal{S}), \lim _{t \rightarrow \infty} x(t)$ exists.

We omit the proof of this result since it is rather technical and it will be developped more widely in a future paper.

4.2. Multi-dimensional case. Our purpose now is to extend the result of Proposition 4.1 to the case of a dimension greater than one. The situation is much more complicated since we have to take into account the geometry of the set $\arg \min G$. In the sequel, we will assume that the gradient of $G$ satisfies the following condition:

$$
\forall \bar{x} \in \operatorname{bd}(\arg \min G), \quad \lim _{x \rightarrow \bar{x}, x \notin \arg \min G} \frac{\nabla G(x)}{|\nabla G(x)|} \quad \text { exists. }
$$

If $G$ is a convex function of a single variable, i.e. $H=\mathbb{R}$, this condition is satisfied when the set $\arg \min G$ is not reduced to a singleton. Before stating the main result 
of nonconvergence for the trajectories of $(\mathcal{S})$, let us first recall some basic notions of convex analysis. The polar cone $K^{*}$ of a cone $K \subset H$ is defined by

$$
K^{*}=\{y \in H \mid \quad \forall x \in K, \quad\langle x, y\rangle \leq 0\} .
$$

Let $S \subset H$ be a closed convex set and let $\bar{x} \in S$. The normal cone $N_{S}(\bar{x})$ and the tangent cone $T_{S}(\bar{x})$ are respectively defined by

$$
\begin{aligned}
& N_{S}(\bar{x})=\{\xi \in H \mid \quad \forall x \in S, \quad\langle\xi, x-\bar{x}\rangle \leq 0\}, \\
& T_{S}(\bar{x})=\operatorname{cl}\left[\bigcup_{\lambda>0} \lambda(S-\bar{x})\right] .
\end{aligned}
$$

The convex cones $N_{S}(\bar{x})$ and $T_{S}(\bar{x})$ are polar to each other, i.e. $N_{S}(\bar{x})=\left[T_{S}(\bar{x})\right]^{*}$ and $T_{S}(\bar{x})=\left[N_{S}(\bar{x})\right]^{*}$. Fur further details relative to convex analysis, the reader is referred to Rockafellar's book [18.

The next lemma brings to light a geometrical property that will be crucial in the proof of the next theorem.

Lemma 4.2. Let $G: H \rightarrow \mathbb{R}$ be a convex function of class $\mathcal{C}^{1}$ such that $S=$ $\arg \min G \neq \emptyset$. Given $\bar{x} \in \operatorname{bd}(S)$, assume that $d:=\lim _{x \rightarrow \bar{x}, x \notin S} \nabla G(x) /|\nabla G(x)|$ exists. Then we have:

(i) $N_{S}(\bar{x})=\mathbb{R}_{+} d$.

(ii) There exists a neighborhood $V$ of $\bar{x}$, a closed convex cone $K \subset H$ along with a positive real $\eta>0$ such that

$$
\forall x \in V, \quad \nabla G(x) \in K \quad \text { and } \quad-K \cap \eta \mathbb{B} \subset S-\bar{x} .
$$

Proof. (i) Since the function $G$ is convex and since $\nabla G(\bar{x})=0$, we have

$$
\forall x \in H, \quad\langle\nabla G(x), x-\bar{x}\rangle \geq 0 .
$$

Let $v \notin T_{S}(\bar{x})$ and take the vector $x$ equal to $\bar{x}+t v$, for some $t>0$. Remark that, since $v \notin T_{S}(\bar{x})$, we have $\bar{x}+t v \notin S$ and hence $\nabla G(\bar{x}+t v) \neq 0$ for every $t>0$. From (31), we derive that

$$
\forall t>0, \quad\left\langle\frac{\nabla G(\bar{x}+t v)}{|\nabla G(\bar{x}+t v)|}, v\right\rangle \geq 0 .
$$

Taking the limit as $t \rightarrow 0^{+}$, we infer that $\langle d, v\rangle \geq 0$. Since this is true for every $v \notin T_{S}(\bar{x})$, we conclude that $-d \in\left[H \backslash T_{S}(\bar{x})\right]^{*}$. Let us denote by $\mathcal{H}_{x, \leq}\left(\right.$ resp. $\left.\mathcal{H}_{x,>}\right)$ the closed (resp. open) hyperplane defined by $\mathcal{H}_{x, \leq}=\{y \in H \mid\langle x, y\rangle \leq 0\}$ (resp. $\left.\mathcal{H}_{x,>}=\{y \in H \mid \quad\langle x, y\rangle>0\}\right)$. The polarity relation $T_{S}(\bar{x})=\left[N_{S}(\bar{x})\right]^{*}$ can be equivalently rewritten as $T_{S}(\bar{x})=\bigcap_{x \in N_{S}(\bar{x})} \mathcal{H}_{x, \leq-}$. Then it follows that

$$
-d \in\left[H \backslash T_{S}(\bar{x})\right]^{*}=\left[\bigcup_{x \in N_{S}(\bar{x})} \mathcal{H}_{x,>}\right]^{*}=\bigcap_{x \in N_{S}(\bar{x})} \mathcal{H}_{x,>}^{*}=\bigcap_{x \in N_{S}(\bar{x})} \mathbb{R}_{-} x .
$$

If the cone $N_{S}(\bar{x})$ is not reduced to a half-line, the above intersection equals $\{0\}$, which contradicts the fact that $|d|=1$. Hence the cone $N_{S}(\bar{x})$ is equal to a half-line and the above inclusion shows that $N_{S}(\bar{x})=\mathbb{R}_{+} . d$.

(ii) Since $\lim _{x \rightarrow \bar{x}, x \notin S} \nabla G(x) /|\nabla G(x)|=d$, there exists a neighborhood $V$ of $\bar{x}$ such that

$$
\forall x \in V, \quad x \notin S \quad \Longrightarrow \quad\left\langle d, \frac{\nabla G(x)}{|\nabla G(x)|}\right\rangle \geq \frac{1}{2} .
$$


Let us define the cone $K=\{0\} \cup\left\{v \in H \mid\left\langle d, \frac{v}{|v|}\right\rangle \geq \frac{1}{2}\right\}$. It is clear that $K$ is a closed convex cone and that $\nabla G(x) \in K$ for every $x \in V$. On the other hand, since $N_{S}(\bar{x})=\mathbb{R}_{+} . d$, we have

$$
\liminf _{v \rightarrow 0, v \notin S-\bar{x}}\left\langle d, \frac{v}{|v|}\right\rangle \geq 0 .
$$

Therefore, there exists $\eta>0$ such that

$$
v \in \eta \mathbb{B} \quad \text { and } \quad v \notin S-\bar{x} \quad \Longrightarrow \quad\left\langle d, \frac{v}{|v|}\right\rangle>-\frac{1}{2} .
$$

Since for every $v \in-K \backslash\{0\},\left\langle d, \frac{v}{|v|}\right\rangle \leq-\frac{1}{2}$, we deduce that $-K \cap \eta \mathbb{B} \subset S-\bar{x}$, which achieves the proof of property $\left(\mathcal{C}_{\bar{x}}\right)$.

Remark 4.1. When the normal cone $N_{S}(\bar{x})$ is reduced to a half-line, the set $S$ is said to be smooth at $\bar{x}$. Lemma 4.2 (i) shows that the existence of $\lim _{x \rightarrow \bar{x}, x \notin S} \nabla G(x) /$ $|\nabla G(x)|$ implies smoothness of the set $S$ at $\bar{x}$. Notice that such a result cannot be obtained by the classical subdifferential calculus for convex functions since the usual qualification conditions fail at $\bar{x}$. However, by using formulas for the subdifferential of the composition of convex functions in terms of the subdifferentials of the data functions at nearby points, one can recoven the expression of $N_{S}(\bar{x})$ given by Lemma 4.2 (i). For more details on sequential convex subdifferential calculus, the reader is referred to 19 .

Let us now state the general result of nonconvergence for the trajectories of $(\mathcal{S})$ under the condition $\int_{0}^{\infty} e^{-\int_{0}^{t} a(s) d s} d t=\infty$.

Theorem 4.1. Let $G: H \rightarrow \mathbb{R}$ be a convex coercive function of class $\mathcal{C}^{1}$ such that $\nabla G$ is Lipschitz continuous on the bounded sets of $H$. Assume moreover that the geometric property (30) holds. Let $a: \mathbb{R}_{+} \rightarrow \mathbb{R}_{+}$be a continuous map such that $\int_{0}^{\infty} e^{-\int_{0}^{t} a(s) d s} d t=\infty$. Given $\left(x_{0}, \dot{x}_{0}\right) \in H^{2}$, consider the unique solution $x$ of $(\mathcal{S})$ satisfying $(x(0), \dot{x}(0))=\left(x_{0}, \dot{x}_{0}\right)$. If $\left(x_{0}, \dot{x}_{0}\right) \notin \arg \min G \times\{0\}$, then the trajectory $x$ of $(\mathcal{S})$ does not converge.

Proof. For simplicity of notation, let us set $S=\arg \min G$. Let us prove the contraposition of the previous statement and assume that there exists $\bar{x} \in H$ such that $\lim _{t \rightarrow \infty} x(t)=\bar{x}$. We must prove that in this case $x_{0} \in S, \dot{x}_{0}=0$. From Proposition 2.5, we have $\nabla G(\bar{x})=0$; hence $\bar{x} \in S$. If $\bar{x} \in \operatorname{bd}(S)$, condition $\left(\mathcal{C}_{\bar{x}}\right)$ is satisfied in view of assumption (30) and Lemma 4.2 (ii). On the other hand, if $\bar{x} \in \operatorname{int}(S)$ condition $\left(\mathcal{C}_{\bar{x}}\right)$ is trivially satisfied with $K=\{0\}$. In both cases, we derive the existence of a closed convex cone $K \subset H$ along with $\eta>0$ and $t_{0} \geq 0$ such that

$$
\forall t \geq t_{0}, \quad \nabla G(x(t)) \in K \quad \text { and } \quad-K \cap \eta \mathbb{B} \subset S-\bar{x} .
$$

Let $v \in-K^{*}$ and take the scalar product of $(\mathcal{S})$ by the vector $v$. Since $\langle\nabla G(x(t)), v\rangle$ $\geq 0$ for every $t \geq t_{0}$, we deduce that

$$
\forall t \geq t_{0}, \quad\langle\ddot{x}(t), v\rangle+a(t)\langle\dot{x}(t), v\rangle \leq 0 .
$$

Let us apply Lemma 4.1(i) to the map $p$ defined by $p(t)=\langle x(t), v\rangle$. Since the trajectory $x$ is bounded, we infer that $\langle\dot{x}(t), v\rangle \geq 0$ for every $t \geq t_{0}$. By integrating on the interval $\left[t, \infty\right.$ [, we find $\langle x(t)-\bar{x}, v\rangle \leq 0$ for every $t \geq t_{0}$. Since this is true for every $v \in-K^{*}$, we derive that $x(t)-\bar{x} \in-K^{* *}$ for every $t \geq t_{0}$. Recalling

\footnotetext{
${ }^{2}$ The first author wishes to thank Lionel Thibault (U. Montpellier II) for this pertinent remark.
} 
that $K^{* *}=K$ for every closed convex cone $K$, we conclude that $x(t)-\bar{x} \in-K$ for every $t \geq t_{0}$. On the other hand, since $\lim _{t \rightarrow \infty} x(t)=\bar{x}$, there exists $t_{1} \geq t_{0}$ such that $x(t)-\bar{x} \in \eta \mathbb{B}$ for every $t \geq t_{1}$. In view of (32), we infer that $x(t) \in S$ for every $t \geq t_{1}$, so that the differential equation $(\mathcal{S})$ becomes

$$
\forall t \geq t_{1}, \quad \ddot{x}(t)+a(t) \dot{x}(t)=0 .
$$

By arguing as in the proof of Lemma 4.1(ii), we deduce that either $\lim _{t \rightarrow \infty}|x(t)|=$ $\infty$ or $x(t)=x\left(t_{1}\right)$ for every $t \geq t_{1}$. Since the map $x$ converges toward $\bar{x}$, the first eventuality does not hold. It follows by backward uniqueness that we have a stationary solution, $x(t)=x_{0}$ for all $t$, which must therefore satisfy $\left(x_{0}, \dot{x}_{0}\right) \in$ $S \times\{0\}$.

\section{The Case of a nonconvex Potential}

In this section, we discuss the case where $G$ is defined on $\mathbb{R}^{n}$ and has multiple critical points, but does not necessarily satisfy condition (11). Instead, we will assume that

(a) $G$ has finitely many critical points $x_{1}, x_{2}, \ldots, x_{N}$.

(b) $G$ attains different values on them; i.e., we can order them such that

$$
\lambda_{1}=G\left(x_{1}\right)<\lambda_{2}=G\left(x_{2}\right)<\lambda_{3}=G\left(x_{3}\right)<\ldots<\lambda_{N}=G\left(x_{N}\right) .
$$

This is the "generic case". We will also use the assumption

(c) $a: \mathbb{R}_{+} \rightarrow \mathbb{R}_{+}$is nonincreasing such that $\int_{0}^{\infty} a(s) d s=\infty$.

Our first result shows that in this case, for each solution there exists exactly one critical point that is visited for arbitrarily long times.

Proposition 5.1. Let $G: \mathbb{R}^{n} \rightarrow \mathbb{R}$ be a coercive function of class $\mathcal{C}^{1}$ such that $\nabla G$ is Lipschitz continuous on the bounded sets of $\mathbb{R}^{n}$. If the assumptions (a)-(c) above are satisfied, then there exists a unique $x^{*} \in\left\{x_{1}, x_{2}, \ldots, x_{N}\right\}$ such that

$$
\lim _{t \rightarrow \infty} \mathcal{E}(t)=G\left(x^{*}\right) \text {. }
$$

Also, for all $T \geq 0$,

$$
\liminf _{t \rightarrow \infty} \sup _{s \in[t, t+T]}\left|x(s)-x^{*}\right|=0 .
$$

Moreover $x^{*}$ is the only point of accumulation that is visited for arbitrarily long time intervals. If $x^{*}$ is a local minimum of $G$, then in fact

$$
\lim _{t \rightarrow \infty}\left|x(t)-x^{*}\right|=0 .
$$

Proof. From Proposition 2.3 , there are arbitrary long time intervals $[S, T]$ where $|\dot{x}(t)|$ is arbitrarily small. Since $t \mapsto \mathcal{E}(t)=\frac{1}{2}|\dot{x}(t)|^{2}+G(x(t))$ is decreasing and bounded below, its limit exists. If this is not equal to $G\left(x_{j}\right)$ for some $j \in\{1, \ldots, N\}$, then there exists an interval $[a, b]$ that does not contain any critical value of $G$, such that $G(x(t)) \in[a, b]$ for every $t \in[S, T]$. Now, we can find $\delta>0$ such that $|g(\xi)| \geq \delta$ on $G^{-1}([a, b])$, and thus $|g(x(t))| \geq \delta$ for every $t \in[S, T]$. However, this contradicts Proposition 2.4. Thus, there exists $x^{*} \in\left\{x_{1}, x_{2}, \ldots, x_{N}\right\}$ such that $\lim _{t \rightarrow \infty} \mathcal{E}(t)=G\left(x^{*}\right)$. Therefore, $\left|x(t)-x^{*}\right|$ becomes arbitrarily small on arbitrarily long intervals by Proposition 2.4. To show that there is no other point $x^{* *}$ such that

$$
\liminf _{t \rightarrow \infty} \sup _{s \in[t, t+T]}\left|x(s)-x^{* *}\right|=0,
$$


firstly suppose that such a point exists with $G\left(x^{* *}\right)=G\left(x^{*}\right)$. Note that then $g\left(x^{* *}\right) \neq 0$. On the other hand, $|\dot{x}(t)|$ must become arbitrarily small on these same intervals, since $\lim _{t \rightarrow \infty} \mathcal{E}(t)=G\left(x^{*}\right)=G\left(x^{* *}\right)$, and therefore also $g(x(t))$ can be made arbitrarily small on such intervals. But this is impossible, since $g\left(x^{* *}\right) \neq 0$.

Next, suppose that $G\left(x^{* *}\right)<G\left(x^{*}\right)$. In this case, we can find arbitrarily long intervals $[S, T]$ where $\left|x(t)-x^{* *}\right|$ is small and therefore $G(x(t))<G\left(x^{*}\right)-\delta$ for some $\delta>0$. Then $|\dot{x}(t)|^{2} \geq 2 \delta$ on these intervals. However, the map $\ddot{x}$ is uniformly bounded on any such interval. By applying Landau's inequality on the interval $[S, T]$

$$
\|\dot{x}\|_{\infty} \leq 2 \sqrt{\left\|x-x^{* *}\right\|_{\infty}\|\ddot{x}\|_{\infty}}
$$

we obtain a contradiction. Hence no such point $x^{* *}$ exists.

Since $\lim _{t \rightarrow \infty} \mathcal{E}(t)=G\left(x^{*}\right)$, the solution $x(t)$ must eventually enter and remain in the connected component of $G^{-1}\left(\left(-\infty, G\left(x^{*}\right)+\varepsilon\right)\right)$ that contains $x^{*}$, for any $\varepsilon>0$. If $x^{*}$ is a local minimum of $G$ (which is strict by assumption), then the intersection of these neighborhoods of $x^{*}$ is just $\left\{x^{*}\right\}$; hence $\lim _{t \rightarrow \infty} x(t)=x^{*}$.

We now give a result that shows that the density of the times $t \in \mathbb{R}_{+}$when $x(t)$ is near the critical point $x^{*}$ approaches 1 . This is comparable to a result on "convergence in probability".

Theorem 5.1. In addition to the hypotheses of Proposition [5.1, we assume that there exists $c>0$ such that $a(t) \geq \frac{c}{t+1}$ for every $t \geq 0$. Then there is a unique stationary point $x^{*} \in\left\{x_{1}, \ldots, x_{N}\right\}$ of $G$ such that for any $\varepsilon>0$,

$$
\lim _{T \rightarrow \infty} T^{-1}\left|\left\{t \leq T|| x(t)-x^{*} \mid>\varepsilon\right\}\right|=0,
$$

where $|A|$ denotes the one-dimensional Lebesgue measure of a measurable set $A \subset$ $\mathbb{R}$.

Proof. We first recall estimate (8), which implies

$$
\int_{0}^{\infty} \frac{1}{t+1}|\dot{x}(t)|^{2} d t<\infty
$$

We are now going to show that

$$
\int_{0}^{\infty} \frac{1}{t+1}|g(x(t))|^{2} d t<\infty
$$

We write $b(t)=\frac{1}{t+1}$. Form the scalar product of $(\mathcal{S})$ with $b(t) g(x(t))$ and integrate over $[0, T]$, for some $T>0$. The result is the identity

$$
\int_{0}^{T} b(t)|g(x(t))|^{2} d t=-\int_{0}^{T} a(t) b(t)\langle\dot{x}(t), g(x(t))\rangle d t-\int_{0}^{T} b(t)\langle\ddot{x}(t), g(x(t))\rangle d t .
$$

Integrating by parts, the first integral on the right hand side becomes

$$
-\int_{0}^{T} a(t) b(t) \frac{d}{d t} G(x(t)) d t=\left.(a(t) b(t) G(x(t)))\right|_{t=T} ^{t=0}+\int_{0}^{T} \frac{d}{d t}(a(t) b(t)) G(x(t)) d t .
$$

Since $G(x(\cdot))$ is bounded on $[0, \infty)$ and $\dot{a} b+a \dot{b}$ is integrable, this term therefore is bounded. The second integral on the right hand side of the above identity becomes, 
after two integrations by parts,

$$
\begin{aligned}
\ldots= & \left.(-b(t)\langle\dot{x}(t), g(x(t))\rangle)\right|_{t=0} ^{t=T}+\int_{0}^{T} \dot{b}(t)\langle\dot{x}(t), g(x(t))\rangle d t \\
& +\int_{0}^{T} b(t)\left\langle\dot{x}(t), \frac{d}{d t} g(x(t))\right\rangle d t \\
= & \left.(-b(t)\langle\dot{x}(t), g(x(t))\rangle+\dot{b}(t) G(x(t)))\right|_{t=0} ^{t=T}-\int_{0}^{T} \ddot{b}(t) G(x(t)) d t \\
& +\int_{0}^{T} b(t)\langle\dot{x}(t), D g(x(t)) \dot{x}(t)\rangle d t,
\end{aligned}
$$

where $D g(x(t))$ is the derivative of $g$ at $x(t)$. The first two terms are both bounded due to previous estimates, and $\|D g(x(t))\| \leq M$ for all $t$ since the trajectory $x(\cdot)$ is bounded and $g=\nabla G$ is Lipschitz, uniformly on bounded sets. Therefore the last integral is bounded in magnitude by

$$
\left|\int_{0}^{T} b(t)\langle\dot{x}(t), D g(x(t)) \dot{x}(t)\rangle d t\right| \leq M \int_{0}^{T} b(t)|\dot{x}(t)|^{2} d t \leq \frac{M}{c} \int_{0}^{T} a(t)|\dot{x}(t)|^{2} d t,
$$

which remains uniformly bounded for all $T>0$. This proves (35).

By Proposition 5.1 $\lim _{t \rightarrow \infty} \mathcal{E}(t)=G\left(x^{*}\right)=\lambda_{i}$ for some stationary point $x^{*}=x_{i}$ of $G$. Pick $\delta>0$ such that $\lambda_{i-1}+\delta<\lambda_{i}<\lambda_{i+1}-\delta$, and let $T_{0}>0$ be so large that $\mathcal{E}(t)<\lambda_{i}+\delta$ if $t \geq T_{0}$. For the remainder of the proof, we assume without loss of generality that $T_{0}=0$. Let $\varepsilon>0$. Then there exists $\gamma>0$ such that

$$
\left|x(t)-x^{*}\right| \geq \varepsilon \Rightarrow|\dot{x}(t)| \geq \sqrt{2 \delta} \quad \text { or } \quad|g(x(t))| \geq \gamma .
$$

Indeed, assume that $\left|x(t)-x^{*}\right| \geq \varepsilon$ and that $|\dot{x}(t)|<\sqrt{2 \delta}$. First we have

$$
G(x(t))=\mathcal{E}(t)-\frac{1}{2}|\dot{x}(t)|^{2} \geq \lambda_{i}-\delta .
$$

Now set

$$
\gamma=\min \left\{|g(\xi)||| \xi-x^{*} \mid \geq \epsilon, \lambda_{i}-\delta \leq G(\xi) \leq \lambda_{i}+\delta\right\} .
$$

The quantity $\gamma$ is positive since there are no critical points of $G$ in the compact region over which $|g(\cdot)|$ is minimized. Hence assertion (36) is proved. Therefore, we deduce that

$$
\begin{aligned}
\left|\left\{t|| x(t)-x^{*} \mid \geq \epsilon\right\}\right| & \leq \mid\{t|| \dot{x}(t) \mid \geq \sqrt{2 \delta} \text { or }|g(x(t))| \geq \gamma\} \mid \\
& \leq|\{t|| \dot{x}(t) \mid \geq \sqrt{2 \delta}\}|+|\{t|| g(x(t)) \mid \geq \gamma\}| .
\end{aligned}
$$

By estimates (34) and (35) combined with Lemma 5.1 below, we derive that

$$
\begin{aligned}
& \lim _{T \rightarrow \infty} T^{-1}\left|\left\{t \leq T|| x(t)-x^{*} \mid \geq \varepsilon\right\}\right| \\
& \quad \leq \lim _{T \rightarrow \infty} T^{-1}|\{t \leq T|| \dot{x}(t) \mid \geq \sqrt{2 \delta}\}|+\lim _{T \rightarrow \infty} T^{-1}|\{t \leq T|| g(x(t)) \mid \geq \gamma\}|=0 .
\end{aligned}
$$

The theorem has been proved.

As a consequence, a Cesaro average of the solution $x(\cdot)$ converges to the critical point $x^{*}$. 
Corollary 5.1. Under the conditions of Theorem [5.1, all solutions of $(\mathcal{S})$ satisfy

$$
\lim _{T \rightarrow \infty} \frac{1}{T} \int_{0}^{T} x(t) d t=x^{*}
$$

for some critical point $x^{*}$ of $G$.

Proof. Let $x^{*}$ be the stationary point identified in Theorem 5.1. Given $\varepsilon>0$ and $T>0$, we have

$$
\left|\frac{1}{T} \int_{0}^{T} x(t) d t-x^{*}\right| \leq \frac{1}{T} \int_{0}^{T}\left|x(t)-x^{*}\right| d t \leq \varepsilon+\frac{M}{T}\left|\left\{t \leq T|| x(t)-x^{*} \mid \geq \varepsilon\right\}\right|,
$$

where $M=\sup _{t \geq 0}\left|x(t)-x^{*}\right|<\infty$. The limsup of the right hand side is no larger than $\varepsilon$, which proves the corollary.

In the same direction, a result of convergence of the ergodic mean has been obtained by Brezis [6] for trajectories associated to semigroups of nonlinear contractions.

Let us now establish a result that was useful in the proof of Theorem 5.1

Lemma 5.1. Let $w:[0, \infty) \rightarrow \mathbb{R}$ be measurable, bounded, and nonnegative. If

$$
\int_{0}^{\infty} \frac{w(t)}{t+1} d t<\infty
$$

then for all $\varepsilon>0$,

$$
\lim _{T \rightarrow \infty} T^{-1}|\{t \leq T \mid w(t)>\varepsilon\}|=0 .
$$

Proof. Let $\varepsilon>0$. For $0 \leq S<T<\infty$, set $A(\varepsilon, S, T)=\{S \leq t \leq T \mid w(t)>\varepsilon\}$. It is clearly sufficient to show that for all $\delta \in(0,1)$ we can find $S>0$ such that

$$
\limsup _{T \rightarrow \infty}(T+1)^{-1}|A(\varepsilon, S, T)| \leq \delta
$$

For this purpose, define $\rho=-\log (1-\delta)$, and choose $S>0$ large enough such that $\int_{S}^{\infty} \frac{w(t)}{t+1} d t \leq \varepsilon \rho$. Then for $T>S$,

$$
\varepsilon \rho \geq \int_{S}^{T} \frac{w(t)}{t+1} d t \geq \int_{A(\varepsilon, S, T)} \frac{\varepsilon}{t+1} d t .
$$

Clearly the integral on the right is minimized if $A(\varepsilon, S, T)=[T-|A(\varepsilon, S, T)|, T]$, and therefore

$$
\rho \geq \int_{T-|A(\varepsilon, S, T)|}^{T} \frac{1}{t+1} d t=-\log \left(1-\frac{|A(\varepsilon, S, T)|}{T+1}\right) .
$$

By the choice of $\rho$, this inequality becomes $\frac{|A(\varepsilon, S, T)|}{T+1} \leq \delta$.

Notice that the above proof mainly relies on a variant of Markov's inequality.

The main remaining open question is whether $\lim _{t \rightarrow \infty} x(t)$ exists if $a(t) \rightarrow 0$ as $t \rightarrow \infty$. There is a unique stationary point of $G$ that is visited for long times. If this point is a local minimum of $G$, the trajectory will converge to it by Proposition 5.1. If the point is a local maximum, it appears possible to adapt the arguments from the next section to show that again convergence holds. The difficulty is that in more than one dimension, the stationary point that is visited for long times may be a saddle point of $G$. Then it is possible that the solution visits other regions of 
$\mathbb{R}^{n}$ intermittently for finite amounts of time, infinitely often, spending longer and longer periods of time near the saddle point in between. In one dimension, such a behavior cannot occur, since solutions either get trapped near local minima of $G$, or if they visit local maxima of $G$ without converging to them, they must leave their neighborhood rapidly.

To end this section, we show that if $a(\cdot)$ is bounded away from $0(\mathrm{e} . \mathrm{g}$. if $a(\cdot)$ is a positive constant), solutions of $(\mathcal{S})$ always converge.

Proposition 5.2. Let $a: \mathbb{R}_{+} \rightarrow \mathbb{R}_{+}$be a nonincreasing map such that $a(t) \geq a_{0}$ for all $t \geq 0$, with some $a_{0}>0$. Let $G: \mathbb{R}^{n} \rightarrow \mathbb{R}$ be a coercive function of class $\mathcal{C}^{1}$, such that $\nabla G$ is Lipschitz continuous on the bounded sets of $\mathbb{R}^{n}$. Assume that $G$ has finitely many critical points $x_{1}, x_{2}, \ldots, x_{N}$. Then, for any solution $x$ to the differential equation $(\mathcal{S})$, there exists $x^{*} \in\left\{x_{1}, x_{2}, \ldots, x_{N}\right\}$ such that $\lim _{t \rightarrow \infty} x(t)=x^{*}$.

Proof. The assumption $a(t) \geq a_{0}>0$ implies that $\int_{0}^{\infty}|\dot{x}(t)|^{2} d t<\infty$ and hence $\lim _{t \rightarrow \infty} \dot{x}(t)=0$, since the map $\ddot{x}$ is uniformly bounded. From Proposition 2.4, we derive that $\lim _{t \rightarrow \infty} g(x(t))=0$. Since the set of zeroes of $g$ is discrete, this implies that $x(t)$ converges to one of the critical points of $G$.

\section{The one-Dimensional CASE}

Let us discuss the asymptotic properties of $(\mathcal{S})$ when $H=\mathbb{R}$. The derivative $\dot{x}$ changes sign either finitely many times or infinitely many times. In the first case, solutions must have a limit, while the second case can occur either if the solution approaches a limit or if the $\omega$-limit of the trajectory is a nonempty interval. We shall give conditions that exclude this last possibility. Rather, trajectories always have a limit, and moreover solutions oscillate infinitely if and only if this limit is a local minimum of $G$. We show further that in this case the set of initial conditions for which solutions converge to a local minimum is open and dense.

To describe the behavior of the trajectories more precisely, let us write

$$
w(t)=\mathcal{E}(t)=G(x(t))+\frac{1}{2}|\dot{x}(t)|^{2}
$$

and observe that

$$
\dot{w}(t)=-a(t)|\dot{x}(t)|^{2}=2 a(t)(G(x(t))-w(t))
$$

and

$$
\dot{x}(t)= \pm \sqrt{2 w(t)-2 G(x(t))} .
$$

Assume that $a(t)>0$ for every $t \geq 0$ and that the solution $x$ is not stationary. It is obvious that $\dot{w}(t)<0$ for $t \geq 0$, except at times $t$ where $w(t)=G(x(t))$, and these $t$ are precisely those times where $\dot{x}$ changes sign. The set $\mathcal{T}=\{t \geq 0 \mid \dot{x}(t)=0\}$ must be discrete. Indeed, if $t^{*}$ is an accumulation point of $\mathcal{T}$, then there exists a sequence $\left(t_{i}\right)$ tending toward $t^{*}$ such that $\dot{x}\left(t_{i}\right)=0$; hence $\dot{x}\left(t^{*}\right)=0$. By Rolle's Theorem, there exists also a sequence $\left(u_{i}\right)$ tending toward $t^{*}$ such that $\ddot{x}\left(u_{i}\right)=0$; hence $\ddot{x}\left(t^{*}\right)=0$. Hence we would have the equality $\dot{x}\left(t^{*}\right)=\ddot{x}\left(t^{*}\right)=G^{\prime}\left(x\left(t^{*}\right)\right)=0$ and thus $x$ would have to be a constant solution, a contradiction. Therefore there exists an increasing sequence $\left(t_{n}\right)$ tending toward $\infty$ such that $\mathcal{T}=\left\{t_{n}, n \in \mathbb{N}\right\}$. As $\mathcal{T}$ is discrete, $w$ is strictly decreasing; hence $\lim _{t \rightarrow \infty} w(t)$ exists if the function $G$ is bounded from below. If $\mathcal{T}$ is finite, i.e. if $\dot{x}$ changes its sign finitely often, then $\lim _{t \rightarrow \infty} x(t)=x^{*}$ exists since $x$ is eventually monotone and is bounded (provided 
some coercivity assumption on $G)$. In this case, $G^{\prime}\left(x^{*}\right)=0$ by Proposition 2.5. However, without additional assumptions on the maps $a$ and $G$, the trajectory $x(\cdot)$ need not converge, as Proposition 4.1 shows.

Before giving the main assumptions of this section, let us recall the definitions of strong convexity and strong concavity.

Definition 6.1. Let $G: \mathbb{R} \rightarrow \mathbb{R}$ be a function of class $\mathcal{C}^{1}$ and let $x^{*} \in \mathbb{R}$. The function $G$ is said to be strongly convex in the neighborhood of $x^{*}$ if there exist $\varepsilon$, $\delta>0$ such that

$$
\forall x, y \in] x^{*}-\varepsilon, x^{*}+\varepsilon\left[, \quad G(y) \geq G(x)+(y-x) G^{\prime}(x)+\delta(y-x)^{2} .\right.
$$

It is easy to check that the above property amounts to saying that the map $x \mapsto G(x)-\delta x^{2}$ is convex on $] x^{*}-\varepsilon, x^{*}+\varepsilon[$. This is also equivalent to the fact that the map $x \mapsto G^{\prime}(x)-2 \delta x$ is nondecreasing on $] x^{*}-\varepsilon, x^{*}+\varepsilon[$. When the function $G$ is of class $\mathcal{C}^{2}$, assertion (37) is equivalent to the inequality $G^{\prime \prime} \geq 2 \delta$ on ]$x^{*}-\varepsilon, x^{*}+\varepsilon[$.

Let us now introduce the notion of strong concavity.

Definition 6.2. Let $G: \mathbb{R} \rightarrow \mathbb{R}$ be a function of class $\mathcal{C}^{1}$ and let $x^{*} \in \mathbb{R}$. The function $G$ is said to be strongly concave in the neighborhood of $x^{*}$ if $-G$ is strongly convex in the neighborhood of $x^{*}$.

We are now able to set up the framework that will be used throughout this section. The function $G: \mathbb{R} \rightarrow \mathbb{R}$ of class $\mathcal{C}^{1}$ will satisfy the following assumptions, considered as the "generic" case.

(a) $G$ has finitely many critical points $x_{1}<x_{k}<\cdots<x_{N}$.

(b) If $k \neq r$, then $G\left(x_{k}\right) \neq G\left(x_{r}\right)$.

(c) For all $k \in\{1, \ldots, N\}$, the function $G$ is either strongly convex or strongly concave in the neighborhood of $x_{k}$.

Property (c) implies that the critical points of $G$ correspond either to local minima or local maxima of $G$. Moreover, property (c) shows that near local minima $x_{j}, G$ satisfies the inequality $G(x) \geq G\left(x_{j}\right)+\delta\left|x-x_{j}\right|^{2}$ and near local maxima $x_{k}$, we have similarly $G(x) \leq G\left(x_{k}\right)-\delta\left|x-x_{k}\right|^{2}$. We can now describe the asymptotic behavior of solutions of $(\mathcal{S})$.

Theorem 6.1. Let $a: \mathbb{R}_{+} \rightarrow \mathbb{R}_{+}$be a differentiable nonincreasing map such that $\lim _{t \rightarrow \infty} a(t)=0$. Assume that there exists $c>0$ such that $a(t) \geq \frac{c}{t+1}$ for every $t \geq 0$. Let $G: \mathbb{R} \rightarrow \mathbb{R}$ be a coercive function of class $\mathcal{C}^{1}$ such that $G^{\prime}$ is Lipschitz continuous on the bounded sets of $\mathbb{R}$. If $G$ satisfies the additional assumptions $(a)-(b)-(c)$ above, then for any solution $x$ of $(\mathcal{S}), \lim _{t \rightarrow \infty} x(t)$ exists. Moreover, denoting by $\mathcal{T}$ the set of sign changes of $\dot{x}$, the limit is a local maximum of $G$ if and only if the set $\mathcal{T}$ is finite, and it is a local minimum of $G$ if and only if $\mathcal{T}$ is infinite.

Proof. We have already observed that if $\mathcal{T}$ is finite, then the trajectory must have a limit and this limit is a critical point of $G$ by Proposition 2.5. Let us now show that the limit is a local maximum of $G$. Arguing by contradiction, let us assume that $\mathcal{T}$ is finite, and that the limit is a local minimum of $G$. Without loss of generality, we may assume that $\lim _{t \rightarrow \infty} x(t)=0$ and that $x$ is nonincreasing for all sufficiently large times. Let $\varepsilon>0$ be such that $g(\xi) \geq 2 \delta \xi$ for $\xi \in] 0, \varepsilon[$ and let $T>0$ be such 
that $0<x(t)<\varepsilon$ for $t>T$. As the map a converges to 0 , one can choose $T$ to get $a(t)<2 \sqrt{\delta}$ for $t>T$. Set $A(t)=\exp \left(\frac{1}{2} \int_{0}^{t} a(s) d s\right)$ and $z(t)=A(t) x(t)$. Then

$$
\ddot{z}(t)+\tilde{g}(t, z(t))=0,
$$

where

$$
\tilde{g}(t, \xi)=A(t) g\left(\frac{\xi}{A(t)}\right)-\frac{a(t)^{2}}{4} \xi-\frac{\dot{a}(t)}{2} \xi .
$$

Recalling that $\dot{a}(t) \leq 0$ for every $t \geq 0$, we obtain $\tilde{g}(t, \xi) \geq \delta \xi$, for $0 \leq \xi \leq A(t) \varepsilon$. We derive that $\ddot{z}(t)=-\tilde{g}(t, z(t)) \leq 0$ and $z$ must be concave down. But since the function $z(t)=A(t) x(t)$ is also positive for $t>T$, it must be increasing for all $t>T$, implying $\ddot{z}(t) \leq-\delta z(t) \leq-\delta z(T)$ for all $t>T$. This contradicts the fact that $z$ remains positive.

We next consider the case where $\mathcal{T}$ is infinite. Without loss of generality, we can assume that $x\left(t_{1}\right)<x\left(t_{2}\right)$, and since $\dot{x}$ changes its sign at each $t_{k}$, one sees that

$$
x\left(t_{2 j-1}\right)<x\left(t_{2 j}\right), \quad x\left(t_{2 j+1}\right)<x\left(t_{2 j}\right)
$$

for all $j \geq 1$. In fact, for all $j$ we have

$$
x\left(t_{2 j-1}\right)<x\left(t_{2 j+1}\right)<x\left(t_{2 j+2}\right)<x\left(t_{2 j}\right) .
$$

Indeed, if e.g. $x\left(t_{2 j+2}\right) \geq x\left(t_{2 j}\right)$ for some $j$, then there exists $s \in\left(t_{2 j+1}, t_{2 j+2}\right)$ (consequently $s>t_{2 j}$ ) satisfying $x(s)=x\left(t_{2 j}\right)$. Thus, since $\dot{x}\left(t_{2 j}\right)=0$, we have $w(s) \geq G(x(s))=G\left(x\left(t_{2 j}\right)\right)=w\left(t_{2 j}\right)$, which is a contradiction to the fact that $w$ is strictly decreasing.

Consequently, $X_{1}=\lim _{j \rightarrow \infty} x\left(t_{2 j+1}\right)$ and $X_{2}=\lim _{j \rightarrow \infty} x\left(t_{2 j}\right)$ both exist, and $X_{1} \leq X_{2}$. We claim that $X_{1}=X_{2}$, which will prove that $\lim _{t \rightarrow \infty} x(t)=X_{1}=X_{2}$ exists. This limit must be a critical point of $G$, by Proposition 2.5. Since we have found a sequence $\left(x\left(t_{k}\right)\right)_{k \geq 0}$ converging to it with $G\left(x\left(t_{k}\right)\right)>G\left(X_{1}\right)=G\left(X_{2}\right)$, the critical point can only be a local minimum, completing the proof of the theorem.

Suppose therefore $X_{1}<X_{2}$. Clearly, $\lim _{t \rightarrow \infty} w(t)=G\left(X_{1}\right)=G\left(X_{2}\right)$ since we have $\dot{x}\left(t_{2 i}\right)=\dot{x}\left(t_{2 i+1}\right)=0$. From Proposition [5.1, there exists a critical point $x^{*}$ of $G$ such that $\lim _{t \rightarrow \infty} w(t)=G\left(x^{*}\right)$. Since the trajectory does not converge, we deduce from Proposition 5.1 that $x^{*}$ is not a local minimum of $G$. Thus in view of assumption (c), $x^{*}$ is a local maximum of $G$. Since $x^{*}$ is an accumulation point of the trajectory $x($.$) , we have x^{*} \in\left[X_{1}, X_{2}\right]$. Observing that the sequence $\left(x\left(t_{2 j+1}\right)\right)_{j \geq 0}$ converges to $X_{1}$ with $G\left(x\left(t_{2 j+1}\right)\right)>G\left(X_{1}\right)$, the point $X_{1}$ cannot be a local maximum of $G$; hence $x^{*} \neq X_{1}$. The same argument shows that $x^{*} \neq X_{2}$, and finally $x^{*} \in\left(X_{1}, X_{2}\right)$. Since $G\left(X_{1}\right)=G\left(X_{2}\right)=G\left(x^{*}\right), X_{1}$ and $X_{2}$ cannot be critical points of $G$ in view of assumption (b). Using the fact that $w$ is nonincreasing, we have $G(\xi) \leq G\left(X_{1}\right)=G\left(X_{2}\right)$ for every $\xi \in\left[X_{1}, X_{2}\right]$. We then deduce that $G^{\prime}\left(X_{1}\right)<0$ and $G^{\prime}\left(X_{2}\right)>0$. We are now in the situation of Lemma 6.1 below, with the limit points $X_{1}$ and $X_{2}$ coinciding respectively with the values defined by (38) and (39). Hence if $t_{i}$ is sufficiently large, then by inequality (41),

$$
t_{i+1} \leq t_{i}+C+C \ln \left(t_{i}+1\right)
$$

with some constant $C$. By induction then

$$
t_{n} \leq C+C n \ln (n+1)
$$


for a suitable positive constant and for all sufficiently large $n$, say $n \geq N$. These estimates imply

$$
\begin{aligned}
\int_{t_{N}}^{\infty} a(t)|\dot{x}(t)|^{2} d t & \geq \sum_{n \geq N} \frac{c}{t_{n+1}+1} \int_{t_{n}}^{t_{n+1}}|\dot{x}(t)|^{2} d t \\
& \geq \sum_{n \geq N} \frac{c D}{t_{n+1}+1} \\
& \geq \sum_{n \geq N} \frac{c D}{1+C+C(n+1) \ln (n+2)}=\infty
\end{aligned}
$$

where (40) in Lemma 6.1 was used to estimate the integrals $\int_{t_{n}}^{t_{n+1}}|\dot{x}(t)|^{2} d t$ from below by $D$. On the other hand, $\int_{t_{N}}^{\infty} a(t)|\dot{x}(t)|^{2} d t$ must be finite. This contradiction proves the theorem.

Remark 6.1 (Assumptions on the map a). A careful examination of the above proof shows that it is possible to slightly weaken the assumption $a(t) \geq \frac{c}{t+1}$ for every $t \geq 0$. In fact, if we merely assume that

$$
\int_{1}^{\infty} a(t \ln t) d t=\infty
$$

then the conclusions of Theorem 6.1 still hold true. We let the reader check that the map $a$ defined by $t \mapsto \frac{1}{(t+1) \ln (\ln (t+3))}$ satisfies the above condition.

Remark 6.2 (Assumptions on the map $G$ ). Assumption (b) can be dropped, at the expense of a more technical proof. If assumption (c) is weakened, the current proof breaks down. For example, if we merely assume that $G(x) \leq G\left(x^{*}\right)-\delta\left|x-x^{*}\right|^{p}$ near local maxima $x^{*}$ with some $p>2$, then we can only show that $t_{n+1} \leq t_{n}+C+$ $C\left(1+t_{n}\right)^{\frac{1}{2}-\frac{1}{p}}$, and stronger assumptions for $a$ must be made, e.g. $a(t) \geq c(1+t)^{-\alpha}$ with $\alpha\left(\frac{3}{2}-\frac{1}{p}\right) \leq 1$. On the other hand, solutions may converge to local minima without oscillating infinitely often, if $G$ is not strongly convex there. For example, $x(t)=(t+1)^{-\beta}$ is a solution of $\ddot{x}(t)+\frac{c}{t+1} \dot{x}(t)+x(t)^{1+\frac{2}{\beta}}=0$, with $\beta>0$ and $c=1+\beta+\beta^{-1}$.

Remark 6.3. Under the assumptions of Theorem 6.1 for any solution of $(\mathcal{S})$ that converges to a local maximum $x^{*}$ of $G$, the set of sign changes $\mathcal{T}=\left\{t_{1}, \ldots, t_{K}\right\}$ is finite. It appears plausible that $K$ and $t_{K}=\max \mathcal{T}$ are bounded in terms of $\mathcal{E}(0)=G(x(0))+\frac{1}{2}|\dot{x}(0)|^{2}$, the potential $G$, and the function $a$.

Let us now establish a result that was used in the proof of Theorem 6.1 Consider a function $G: \mathbb{R} \rightarrow \mathbb{R}$ as in Theorem 6.1 and let $x^{*}$ be a local maximum of $G$. Let us define $X_{1}, X_{2}$ respectively by

$$
X_{1}=\sup \left\{x \leq x^{*} \mid G(x)>G\left(x^{*}\right)\right\}
$$

and

$$
X_{2}=\inf \left\{x \geq x^{*} \mid G(x)>G\left(x^{*}\right)\right\} .
$$

The coercivity of $G$ shows that $-\infty<X_{1} \leq X_{2}<\infty$. Since $x^{*}$ is a local maximum, it is clear that $X_{1}<x^{*}<X_{2}$. The continuity of $G$ shows that $G\left(X_{1}\right)=G\left(X_{2}\right)=$ $G\left(x^{*}\right)$. In view of assumption (b), this implies that $X_{1}$ and $X_{2}$ are not critical 
points of $G$. Since $G(x) \leq G\left(X_{1}\right)=G\left(X_{2}\right)$ for every $x \in\left[X_{1}, X_{2}\right]$, we then have $G^{\prime}\left(X_{1}\right)<0$ and $G^{\prime}\left(X_{2}\right)>0$.

Lemma 6.1. Let $a: \mathbb{R}_{+} \rightarrow \mathbb{R}_{+}$and $G: \mathbb{R} \rightarrow \mathbb{R}$ be as in Theorem 6.1. Let $x^{*}$ be a local maximum of $G$ and let $X_{1}, X_{2}$ be the real numbers respectively defined by (38) and (39). Let $x(\cdot)$ be a solution of $(\mathcal{S})$ and let $\mathcal{T}=\left\{t_{i} \mid i \geq 1\right\}$ be the set of sign changes of $\dot{x}$. Assume that for some $i \geq 1, x\left(t_{i}\right)<X_{1}<X_{2}<x\left(t_{i+1}\right)$ and $G^{\prime}$ is negative on $\left[x\left(t_{i}\right), X_{1}\right]$ and positive on $\left[X_{2}, x\left(t_{i+1}\right)\right]$. Then there exist $C>0, D>0$, and $T_{0}>0$ that depend only on $G, a$, and the initial data such that if $t_{i} \geq T_{0}$, then

$$
\begin{aligned}
\int_{t_{i}}^{t_{i+1}}|\dot{x}(t)|^{2} d t & \geq D, \\
t_{i+1}-t_{i} & \leq C+C \ln \left(1+t_{i}\right) .
\end{aligned}
$$

These conclusions also hold true in the symmetric situation corresponding to $x\left(t_{i+1}\right)$ $<X_{1}<X_{2}<x\left(t_{i}\right)$.

Proof. The assumption $x\left(t_{i}\right)<x\left(t_{i+1}\right)$ implies $\dot{x}>0$ on $\left(t_{i}, t_{i+1}\right)$. Since $G$ is strongly concave near $x^{*}$, there exist $\varepsilon, \delta>0$ such that $G(x) \leq G\left(x^{*}\right)-$ $\delta\left|x-x^{*}\right|^{2}$ whenever $\left|x-x^{*}\right| \leq \varepsilon$. We can assume that $x^{*}=0, G\left(x^{*}\right)=0$ and that $X_{1}<-\varepsilon<\varepsilon<X_{2}$. Define numbers $\tau, s, s+h, \tau^{\prime} \in\left(t_{i}, t_{i+1}\right)$ by the conditions $x(\tau)=X_{1}, x(s)=-\varepsilon, x(s+h)=\varepsilon, x\left(\tau^{\prime}\right)=X_{2}$. We first prove inequality (40). Recalling that $w(t) \geq w\left(t_{i+1}\right)=G\left(x\left(t_{i+1}\right)\right)>0$ for every $t \in\left[t_{i}, t_{i+1}\right]$, we have

$$
\begin{aligned}
\int_{t_{i}}^{t_{i+1}}|\dot{x}(t)|^{2} d t & =\int_{t_{i}}^{t_{i+1}} \dot{x}(t) \sqrt{2 w(t)-2 G(x(t))} d t \\
& \geq \int_{\tau}^{\tau^{\prime}} \dot{x}(t) \sqrt{-2 G(x(t))} d t \\
& =\int_{X_{1}}^{X_{2}} \sqrt{-2 G(x)} d x=D>0 .
\end{aligned}
$$

We next prove inequality (41). We first show that

$$
\begin{gathered}
s-t_{i} \leq C, \\
h \leq C+C \ln \left(1+t_{i+1}\right),
\end{gathered}
$$

and

$$
t_{i+1}-(s+h) \leq C .
$$

We claim that we can find $c>0$ such that for sufficiently large $t_{i}$,

$$
w(t) \geq G\left(x\left(t_{i}\right)\right)-c\left(x(t)-x\left(t_{i}\right)\right) \geq G(x(t))
$$

for $t \in\left[t_{i}, \tau\right]$. Indeed, choose $0<c<-\max _{\left[x\left(t_{i}\right), X_{1}\right]} G^{\prime}$. Then $G(x)-G\left(x\left(t_{i}\right)\right) \leq$ $-c\left(x-x\left(t_{i}\right)\right)$ for $x \in\left(x\left(t_{i}\right), X_{1}\right)$. Now define

$$
T_{0}=\inf \{s \geq 0 \mid a(s) \sqrt{2 w(0)-2 \min G} \leq c\} .
$$

Since $\dot{w}(t)=-a(t)|\dot{x}(t)|^{2}$ and $|\dot{x}(t)| \leq \sqrt{2 w(0)-2 \min G}$ for every $t \geq 0$, the inequality $\dot{w}(t) \geq-c|\dot{x}(t)|$ then holds for all $t \geq T_{0}$. An integration then shows 
immediately that $w(t) \geq G\left(x\left(t_{i}\right)\right)-c\left(x(t)-x\left(t_{i}\right)\right)$ for $t \in\left[t_{i}, t_{i+1}\right]$ if $t_{i} \geq T_{0}$. For such $t_{i}$,

$$
\begin{aligned}
\tau-t_{i} & =\int_{t_{i}}^{\tau} \frac{\dot{x}(t)}{\dot{x}(t)} d t=\int_{t_{i}}^{\tau} \frac{\dot{x}(t)}{\sqrt{2 w(t)-2 G(x(t))}} d t \\
& \leq \int_{t_{i}}^{\tau} \frac{\dot{x}(t)}{\sqrt{2 G\left(x\left(t_{i}\right)\right)-2 c\left(x(t)-x\left(t_{i}\right)\right)-2 G(x(t))}} d t \\
& =\int_{x\left(t_{i}\right)}^{X_{1}} \frac{d x}{\sqrt{2 G\left(x\left(t_{i}\right)\right)-2 c\left(x-x\left(t_{i}\right)\right)-2 G(x)}} .
\end{aligned}
$$

The term under the square root is equivalent to $-2\left(G^{\prime}\left(x\left(t_{i}\right)\right)+c\right)\left(x-x\left(t_{i}\right)\right)$ as $x \rightarrow x\left(t_{i}\right)$ and it ensues that the above integral is convergent, due to the choice of $c$. Therefore, we derive that $\tau-t_{i} \leq C_{1}$ for some constant $C_{1}$ that depends on $G$ and $c$. We next estimate $s-\tau$. Note that by construction, $G(x)<0$ on $\left(X_{1},-\varepsilon\right]$ and $G^{\prime}\left(X_{1}\right)<0$. Hence $x \mapsto \frac{1}{\sqrt{-G(x)}}$ is integrable on $\left(X_{1},-\varepsilon\right]$. Then as before

$$
\begin{aligned}
s-\tau & =\int_{\tau}^{s} \frac{\dot{x}(t)}{\sqrt{2 w(t)-2 G(x(t))}} d t \\
& \leq \int_{\tau}^{s} \frac{\dot{x}(t)}{\sqrt{-2 G(x(t))}} d t \\
& =\int_{X_{1}}^{-\varepsilon} \frac{d x}{\sqrt{-2 G(x)}} \\
& =C_{2}
\end{aligned}
$$

for another constant $C_{2}$. These two estimates imply (42). Note that these two estimates do not use any information about $G$ on $\left[-\varepsilon, x\left(t_{i+1}\right)\right]$, and they hold also if in fact $x(\cdot)$ is monotone on $\left[t_{i}, \infty\right)$ and converges to $x^{*}$. That is,

$$
s=\inf \left\{t>t_{i} \mid x(t) \geq-\varepsilon\right\} \leq t_{i}+C
$$

for some $C$ that depends only on $G$ and $a$.

Next, let us show (44), employing the same argument that was used to show (42):

$$
\begin{aligned}
t_{i+1}-(s+h) & =\int_{s+h}^{t_{i+1}} \frac{\dot{x}(t)}{\sqrt{2 w(t)-2 G(x(t))}} d t \\
& \leq \int_{s+h}^{t_{i+1}} \frac{\dot{x}(t)}{\sqrt{2 G\left(x\left(t_{i+1}\right)\right)-2 G(x(t))}} d t \\
& =\int_{\varepsilon}^{x\left(t_{i+1}\right)} \frac{d x}{\sqrt{2 G\left(x\left(t_{i+1}\right)\right)-2 G(x)}} \\
& =C_{3},
\end{aligned}
$$

where $C_{3}$ depends on $G$ and $\varepsilon$. 
We now show (43). Recall that $G(x) \leq-\delta|x|^{2}$ on the interval $[-\varepsilon, \varepsilon]$ and that $w(t) \geq w(s+h)$ for every $t \in[s, s+h]$. Then

$$
\begin{aligned}
h & =\int_{s}^{s+h} \frac{\dot{x}(t)}{\sqrt{2 w(t)-2 G(x(t))}} d t \\
& \leq \int_{s}^{s+h} \frac{\dot{x}(t)}{\sqrt{2 w(s+h)+2 \delta|x(t)|^{2}}} d t \\
& =\int_{-\varepsilon}^{\varepsilon} \frac{d x}{\sqrt{2 w(s+h)+2 \delta x^{2}}} \\
& =\sqrt{\frac{2}{\delta}}\left[\ln \left(x+\sqrt{x^{2}+\frac{w(s+h)}{\delta}}\right)\right]_{0}^{\varepsilon} \\
& \leq C_{4}-C_{5} \ln w(s+h),
\end{aligned}
$$

for suitable constants $C_{4}, C_{5}$ that depend on $\varepsilon$ and $\delta$. Let us estimate the quantity $w(s+h)$ :

$$
\begin{aligned}
w(s+h) & =w\left(t_{i+1}\right)+\int_{s+h}^{t_{i+1}} a(t)|\dot{x}(t)|^{2} d t \\
& \geq \int_{s+h}^{t_{i+1}} \frac{c}{t+1}|\dot{x}(t)|^{2} d t \geq \frac{c}{t_{i+1}+1} \int_{s+h}^{t_{i+1}}|\dot{x}(t)|^{2} d t .
\end{aligned}
$$

By arguing as in the proof of inequality (40), it is immediate to check that

$$
\int_{s+h}^{t_{i+1}}|\dot{x}(t)|^{2} d t \geq \int_{\varepsilon}^{X_{2}} \sqrt{-2 G(x)} d x=D^{\prime}>0 .
$$

It ensues that $w(s+h) \geq \frac{c D^{\prime}}{t_{i+1}+1}$ and consequently

$$
h \leq C+C \ln \left(t_{i+1}+1\right)
$$

for some $C>0$. Combining (42), (43) and (44) results in

$$
t_{i+1}-t_{i} \leq C+C \ln \left(1+t_{i+1}\right)
$$

and therefore by an elementary argument

$$
t_{i+1}-t_{i} \leq C+C \ln \left(1+t_{i}\right)
$$

with some new constant $C>0$. This proves (41) completely.

Remark 6.4. By combining (43) and (44), we obtain

$$
t_{i+1}-s \leq C+C \ln \left(1+t_{i+1}\right)
$$

and therefore by the same argument as above,

$$
t_{i+1}-s \leq C+C \ln (1+s),
$$

with some new constant $C>0$. Note that the proof of (46) does not use any assumptions about the behavior of $x(\cdot)$ for $t<s$.

We now show that under the assumptions of Theorem 6.1, solutions generically converge to a local minimum of $G$.

Theorem 6.2. Under the assumptions of Theorem 6.1, the set of initial data $\left(x_{0}, x_{1}\right)$ for which $\lim _{t \rightarrow \infty} x(t)$ is a local minimum of $G$ is open and dense. 
Proof. For $T>0$, define the map $F_{T}: \mathbb{R}^{2} \rightarrow \mathbb{R}^{2}$ as $F_{T}(u, v)=(x(T), \dot{x}(T))$, where $x$ is the solution of $(\mathcal{S})$ with $x(0)=u, \dot{x}(0)=v$. By standard results for ordinary differential equations, see e.g. [13, $F_{T}$ is a diffeomorphism and has an inverse $F_{-T}$. The inverse diffeomorphism maps $(u, v)=(x(T), \dot{x}(T))$ to $F_{-T}(u, v)=(x(0), \dot{x}(0))$ by solving $(\mathcal{S})$ backwards on $[0, T]$.

Let $x$ be a solution for which $\lim _{t \rightarrow \infty} x(t)=\bar{x}$ is a local minimum of $G$, with $x(0)=x_{0}$ and $\dot{x}(0)=x_{1}$. We shall find a neighborhood of $\left(x_{0}, x_{1}\right)$ such that solutions with initial data from this neighborhood have the same limit. There exist an open interval $I$ containing $\bar{x}$ and $\delta>0$ such that $\bar{x}$ is the only minimum of $G$ in $I$ and $I$ is one of the connected components of $G^{-1}([G(\bar{x}), G(\bar{x})+\delta))$. There is a time $T>0$ such that $x(t) \in I$ and $w(t)=G(x(t))+\frac{1}{2}|\dot{x}(t)|^{2}<G(\bar{x})+\delta$ for $t>T$. Consider the open set $\mathcal{O}=\{(u, v)|u \in I| v \mid,<\sqrt{2 \delta+2 G(\bar{x})-2 G(u)}\}$. By construction this set contains $(x(T), \dot{x}(T))$. Any solution $y$ of $(\mathcal{S})$ with data $(y(T), \dot{y}(T)) \in \mathcal{O}$ satisfies

$$
\forall t \geq T \quad G(y(t)) \leq w(t) \leq w(T) \leq G(\bar{x})+\delta .
$$

Using the definition of $\delta$ and $I$, we conclude that $y$ stays in $I$ for all times greater than $T$. Since $I$ contains only one critical point of $G$ which is a local minimum, we infer that $y(t) \rightarrow \bar{x}$ as $t \rightarrow \infty$. Then $F_{-T}(\mathcal{O})$ is an open neighborhood containing $\left(x_{0}, x_{1}\right)$, and all trajectories with initial data in $F_{-T}(\mathcal{O})$ also converge to $\bar{x}$.

Next let $\mathcal{I}$ be the set of initial data in $\mathbb{R}^{2}$ whose solutions converge to a local maximum of $G$. We must show that it has empty interior. We first show the following:

Claim 6.1. Let $x$ be a solution with initial data $(x(0), \dot{x}(0)) \in \mathcal{I}$ and let $x^{*}$ be the limit of $x(t)$ as $t \rightarrow \infty$. In any neighborhood $\mathcal{U}$ of $(x(0), \dot{x}(0))$, there exist $\left(y_{0}, y_{1}\right)$ such that the corresponding solution $y$ of $(\mathcal{S})$ satisfies $y^{*}=\lim _{t \rightarrow \infty} y(t) \neq x^{*}$ and $G\left(y^{*}\right)<G\left(x^{*}\right)$.

Let $\bar{\lambda}=\min \left\{G(z) \mid g(z)=0, G(z)>G\left(x^{*}\right)\right\}$ be the next smallest critical value of $G$. Since $G$ is strictly concave near $x^{*}$, we can find an interval $I=\left[x^{*}-\varepsilon, x^{*}+\varepsilon\right]$ such that $g$ is strictly decreasing on $I$. Let $T \geq 0$ be such that $\left|x(T)-x^{*}\right|=\varepsilon$ and $\dot{x}$ does not change sign on $[T, \infty)$. After reducing $\varepsilon$, we may assume that $w(T)<\bar{\lambda}$. After replacing $x$ with $x^{*}-x$ and $G(\xi)$ with $G\left(x^{*}-\xi\right)$ if necessary, we may also assume that $x(T)=x^{*}-\varepsilon<x(t)<x^{*}$ and $\dot{x}(t)>0$ for $t>T$. We claim that there exists a nonempty open interval $J$ containing $\dot{x}(T)$ such that whenever $y$ is a solution of $(\mathcal{S})$ with $y(T)=x(T)$ and $\dot{y}(T) \in J, \dot{y}(T) \neq \dot{x}(T)$, then $y^{*}=\lim _{t \rightarrow \infty} y(t) \neq x^{*}$ and $G\left(y^{*}\right)<G\left(x^{*}\right)$.

Indeed, first assume that there exists a solution $y \neq x$ with $y(T)=x(T)$ that converges to $x^{*}$, such that $\dot{y}(t)>0$ for $t>T$. Then $v=x-y$ satisfies

$$
\ddot{v}(t)+a(t) \dot{v}(t)+g(y(t)+v(t))-g(y(t))=0
$$

as well as $v(T)=0, \lim _{t \rightarrow \infty} v(t)=0$. If $v$ has a positive maximum at some $t^{*}>T$, then $\dot{v}\left(t^{*}\right)=0$ and $\ddot{v}\left(t^{*}\right) \leq 0$; hence $g\left(y\left(t^{*}\right)+v\left(t^{*}\right)\right)-g\left(y\left(t^{*}\right)\right)=g\left(x\left(t^{*}\right)\right)-g\left(y\left(t^{*}\right)\right) \geq$ 0 . But we have $g\left(x\left(t^{*}\right)\right)<g\left(y\left(t^{*}\right)\right)$ since $x^{*}-\varepsilon \leq y\left(t^{*}\right)<x\left(t^{*}\right) \leq x^{*}$ and since the map $g$ is decreasing on $\left[x^{*}-\varepsilon, x^{*}\right]$, a contradiction. The same argument applies if $v\left(t^{* *}\right)$ is a negative minimum of $v$. So for any solution $y \neq x$ of $(\mathcal{S})$ with $y(T)=x(T)$ that converges to $x^{*}$, the derivative $\dot{y}$ must have at least one change of sign.

Next let us assume that there is a sequence of solutions $y_{k}$ such that $y_{k}(T)=$ $x(T), \lim _{k \rightarrow \infty} \dot{y}_{k}(T)=\dot{x}(T)$, and $\lim _{t \rightarrow \infty} y_{k}(t)=x^{*}$ for all $k$. By the previous 
argument, the derivatives $\dot{y}_{k}$ must all change sign at least once on $(T, \infty)$. That is, for each $k$ there exists some minimal $t_{k}>T$ such that $\dot{y}_{k}\left(t_{k}\right)=0$. Then $G\left(y_{k}\left(t_{k}\right)\right)>G\left(x^{*}\right)$ and hence $y_{k}\left(t_{k}\right)>x^{*}+\varepsilon$. Let $T_{k} \in\left(T, t_{k}\right)$ be such that $y_{k}\left(T_{k}\right)=x^{*}+\varepsilon$. By Remark 6.4 after Lemma 6.1, especially inequality (46), we see that $T_{k}<t_{k} \leq T+C+C \ln (1+T)$ for all $k \in \mathbb{N}$, for some constant $C$. By standard results on the continuous dependence of solutions of ordinary differential equations on initial data, we have

$$
\lim _{k \rightarrow \infty} \sup _{t \in[T, S]}\left|y_{k}(t)-x(t)\right|=0
$$

for any $S \geq T$. Recalling that $x(t)<x^{*}$ for every $t \geq T$, we have $\left|y_{k}\left(T_{k}\right)-x\left(T_{k}\right)\right|>$ $\varepsilon$, which contradicts formula (47) applied with $S=T+C+C \ln (1+T)$. The contradiction shows that for some open interval $J$ containing $\dot{x}(T)$, solutions $y$ of $(\mathcal{S})$ with $y(T)=x(T)$ and $\dot{y}(T) \in J, \dot{y}(T) \neq \dot{x}(T)$ always have limits $y^{*} \neq x^{*}$. By shrinking the interval $J$ if necessary, we can guarantee that $G\left(y^{*}\right)<\bar{\lambda}$ and therefore also $G\left(y^{*}\right)<G\left(x^{*}\right)$ for all such solutions. Consider then the set $F_{-T}(\{x(T)\} \times J)$. It contains initial data arbitrarily close to $(x(0), \dot{x}(0))$ whose solutions converge to a limit $y^{*}$ with $G\left(y^{*}\right)<G\left(x^{*}\right)$. This proves Claim 6.1.

To complete the proof of the theorem, let $n \geq 0$ be the number of local maxima of $G$. If $G$ has no local maximum, the set $\mathcal{I}$ is empty. Let us now assume that $n \geq 1$. Let $X_{0}=(x(0), \dot{x}(0)) \in \mathcal{I}$ and let us denote by $x^{*}$ the limit of the corresponding solution $x$ of $(\mathcal{S})$. Let us fix some $\varepsilon>0$. From Claim 6.1, there exists $Y_{0}^{(1)} \in \mathbb{R}^{2}$ such that $\left|Y_{0}^{(1)}-X_{0}\right|<\varepsilon / n$ and such that the limit $y^{(1), *}$ of the corresponding solution of $(\mathcal{S})$ satisfies $G\left(y^{(1), *}\right)<G\left(x^{*}\right)$. If $Y_{0}^{(1)} \in \mathcal{I}$, we can again apply Claim 6.1. In fact, a repeated application of Claim 6.1 shows that there exist $k \leq n$ along with $Y_{0}^{(2)}, \ldots, Y_{0}^{(k)} \in \mathbb{R}^{2}$ such that $Y_{0}^{(k)} \notin \mathcal{I}$ and

$$
\forall i \in\{1, \ldots, k-1\}, \quad Y_{0}^{(i)} \in \mathcal{I} \quad \text { and } \quad\left|Y_{0}^{(i+1)}-Y_{0}^{(i)}\right| \leq \varepsilon / n .
$$

By summation, we derive that $\left|Y_{0}^{(k)}-X_{0}\right| \leq \frac{k \varepsilon}{n} \leq \varepsilon$. Since the existence of such a point $Y_{0}^{(k)} \notin \mathcal{I}$ is satisfied for every $\varepsilon>0$ and every $X_{0} \in \mathcal{I}$, we conclude that $\mathcal{I}$ has empty interior.

\section{Appendix A: Stochastic approximation of $(\mathcal{S})$}

We show in this appendix how the stochastic approximation scheme (4) defined in the introduction naturally yields an ordinary differential equation of type $(\mathcal{S})$. Using the same notation as in the introductory paragraph, we define $h^{n+1}$ as the average drift at step $n$ by

$$
h^{n+1}=\frac{\sum_{i=0}^{n} \varepsilon_{i} g\left(X^{i}, \omega^{i+1}\right)}{\sum_{i=0}^{n} \varepsilon_{i}}
$$


and we set $\tau^{n}=\varepsilon_{0}+\varepsilon_{1}+\cdots+\varepsilon_{n}$. We can then rewrite (4) in

$$
\left\{\begin{array}{l}
\left(X^{0}, \tau^{0}, h^{0}\right) \in \mathbb{R}^{d} \times\left\{\varepsilon_{0}\right\} \times\{0\}, \\
\tau^{n+1}=\tau^{n}+\varepsilon_{n+1} \quad \forall n \in \mathbb{N}, \\
h^{n+1}=h^{n}-\varepsilon_{n} \frac{h^{n}}{\tau^{n}}+\varepsilon_{n} \frac{g\left(X^{n}, \omega^{n+1}\right)}{\tau^{n}} \quad \forall n \in \mathbb{N}, \\
X^{n+1}=X^{n}-\varepsilon_{n+1} h^{n+1} \quad \forall n \in \mathbb{N} .
\end{array}\right.
$$

The recursion (48) can be identified now as

$$
\left(\tau^{n+1}, h^{n+1}, X^{n+1}\right)=\left(\tau^{n}, h^{n}, X^{n}\right)-\varepsilon_{n+1} H\left(\tau^{n}, h^{n}, X^{n}, \omega^{n+1}\right)+\varepsilon_{n+1} \eta^{n+1},
$$

where $H(\tau, h, X, \omega)=(-1,(h-g(X, \omega)) / \tau, h)$ and the residual perturbation $\eta^{n+1}$ is given by

$$
\eta^{n+1}=\left(0,\left(\varepsilon_{n+1}-\varepsilon_{n}\right) \frac{h^{n}-g\left(X^{n}, \omega^{n+1}\right)}{\tau^{n} \varepsilon_{n+1}}, \varepsilon_{n} \frac{h^{n}-g\left(X^{n}, \omega^{n+1}\right)}{\tau^{n}}\right) .
$$

Remark then that $\eta^{n} \rightarrow 0$ as $n \rightarrow \infty$ and the conditional expectation with respect to the filtration $\mathcal{F}_{n}$ of $H\left(\tau^{n}, h^{n}, X^{n}, \omega^{n+1}\right)$ is

$$
\mathbb{E}\left[H\left(\tau^{n}, h^{n}, X^{n}, \omega^{n+1}\right) \mid \mathcal{F}_{n}\right]=\left(-1, \frac{h^{n}-g\left(X^{n}\right)}{\tau^{n}}, h^{n}\right) .
$$

Applying the result of [17, the time interpolation of the process $\left(\tau^{n}, h^{n}, X^{n}\right)_{n \geq 1}$ asymptotically behaves as the solution of the following system of differential equations:

$$
\left\{\begin{array}{l}
\dot{\tau}(t)=1 \quad \forall t \in \mathbb{R}, \\
\dot{h}(t)=-\frac{h(t)-g(X(t))}{\tau(t)} \quad \forall t \in \mathbb{R}, \\
\dot{X}(t)=-h(t) \quad \forall t \in \mathbb{R} .
\end{array}\right.
$$

If we set $\beta=\tau(0)$, we derive that the map $X$ satisfies the following o.d.e.:

$$
\ddot{X}(t)=-\frac{\dot{X}(t)+g(X(t))}{t+\beta},
$$

as announced in the introduction.

\section{Appendix B: Special Cases}

Consider first the equation

$$
\ddot{x}(t)+\frac{c}{t} \dot{x}(t)+x(t)=0
$$

for $t>0$ and its shifted version

$$
\ddot{x}(t)+\frac{c}{t+1} \dot{x}(t)+x(t)=0 .
$$

Let $\tilde{x}$ be a solution of the Bessel equation

$$
t^{2} \ddot{x}(t)+t \dot{x}(t)+\left(t^{2}-\left(\frac{c-1}{2}\right)^{2}\right) x(t)=0
$$


for $t>0$, i.e.

$$
\tilde{x}(t)=b_{1} J_{(c-1) / 2}(t)+b_{2} Y_{(c-1) / 2}(t),
$$

where $J_{(c-1) / 2}$ and $Y_{(c-1) / 2}$ are Bessel functions of the first and second kind. A calculation shows that

$$
x(t)=t^{\frac{1-c}{2}} \tilde{x}(t)
$$

is a solution of (50). For $x(0)$ to be finite, we require $b_{2}=0$. Hence the general solution of (50) with finite $x(0)$ is

$$
x_{c}(t)=b_{1} t^{\frac{1-c}{2}} J_{(c-1) / 2}(t) .
$$

Since $J_{(c-1) / 2}(t) \approx \sqrt{\frac{2}{\pi t}} \cos \left(t-\frac{c \pi}{4}\right)$ as $t \rightarrow \infty$ for all $c$, we therefore see that

$$
x_{c}(t) \approx C t^{-c / 2} \cos \left(t-\frac{c \pi}{4}\right)
$$

as $t \rightarrow \infty$, with a suitable constant $C$.

Solutions of (51) are of the form

$$
x(t)=b_{1}(t+1)^{\frac{1-c}{2}} J_{(c-1) / 2}(t+1)+b_{2}(t+1)^{\frac{1-c}{2}} Y_{(c-1) / 2}(t+1)
$$

and have the asymptotic behavior

$$
x(t) \approx t^{-c / 2}\left(C \cos \left(t-\varphi_{0}\right)\right)
$$

with a suitable amplitude constant $C$ and phase shift $\varphi_{0}$. This is the typical behavior of solutions of $(\mathcal{S})$ in one dimension near nondegenerate local minima of $G$, in the case $a(t)=c t^{-1}$ or $a(t)=c(t+1)^{-1}$.

Consider now the equation

$$
\ddot{y}(t)+\frac{c}{t} \dot{y}(t)-y(t)=0
$$

for $t>0$ and its shifted version

$$
\ddot{y}(t)+\frac{c}{t+1} \dot{y}(t)-y(t)=0 .
$$

We are interested in solutions $y_{c}$ that converge to 0 . Let $\tilde{y}$ be a solution of the modified Bessel equation

$$
t^{2} \ddot{y}(t)+t \dot{y}(t)-\left(t^{2}+\left(\frac{c-1}{2}\right)^{2}\right) y(t)=0
$$

for $t>0$, i.e.

$$
\tilde{y}(t)=b_{1} I_{(c-1) / 2}(t)+b_{2} K_{(c-1) / 2}(t),
$$

where $I_{(c-1) / 2}$ and $K_{(c-1) / 2}$ are modified Bessel functions of the first and second kind. A direct calculation shows again that

$$
y_{c}(t)=t^{\frac{1-c}{2}} \tilde{y}(t)
$$

is a solution of (52). For $y_{c}$ to be convergent to 0 , we require $b_{1}=0$, since $I_{\nu}(t) \rightarrow \infty$ as $t \rightarrow \infty$. Thus solutions of (52) that converge to zero are of the form

$$
y_{c}(t)=b_{2} t^{\frac{1-c}{2}} K_{(c-1) / 2}(t) .
$$

Since $K_{(c-1) / 2}(t) \approx \sqrt{\frac{2}{\pi t}} e^{-t}$ for all $c$ as $t \rightarrow \infty$ with higher order terms depending on $c$, we see that

$$
y_{c}(t) \approx C t^{-c / 2} e^{-t}
$$


as $t \rightarrow \infty$, with some constant $C$. Solutions of (53) that converge to 0 then are of the form

$$
y(t)=b_{2}(t+1)^{\frac{1-c}{2}} K_{(c-1) / 2}(t+1)
$$

and have the same asymptotic behavior. This is the typical behavior of solutions of $(\mathcal{S})$ in one dimension near nondegenerate local maxima of $G$, again in the case $a(t)=c t^{-1}$ or $a(t)=c(t+1)^{-1}$. The standard reference for results on Bessel functions is [1].

\section{Note ADDED IN PROOF}

Since this paper was submitted, the authors have learned that a version of Theorem 6.1 in the case $a(t)=\frac{k}{t}$ was proved in [14], with a very similar proof.

\section{REFERENCES}

[1] M. Abramowitz, I. Stegun, Handbook of Mathematical Functions. Reprint of the 1972 edition, Dover, New York, 1992. MR.1225604 (94b:00012)

[2] F. Alvarez, On the minimizing property of a second order dissipative system in Hilbert spaces, SIAM J. on Control and Optimization, 38 (2000), $\mathrm{n}^{\circ} 4,1102-1119$. MR1760062(2001e:34118)

[3] H. Attouch, R. Cominetti, A dynamical approach to convex minimization coupling approximation with the steepest descent method, J. Differential Equations, 128 (1996), 519-540. MR1398330 (97e:90112)

[4] H. Attouch, M.-O. Czarnecki, Asymptotic control and stabilization of nonlinear oscillators with non isolated equilibria , J. Differential Equations, 179 (2002), 278-310. MR.1883745 (2004a:93068)

[5] H. Attouch, X. Goudou, P. Redont, The heavy ball with friction method: I. The continuous dynamical system, Communications in Contemporary Mathematics, 2 (2000), $\mathrm{n}^{\circ}$ 1, 1-34. MR1753136 (2001b:37025)

[6] H. Brezis, Asymptotic behavior of some evolution systems. In: Nonlinear Evolution Equations (Proc. Sympos., Univ. Wisconsin, Madison, Wis., 1977), 141-154. Academic Press, New York-London, 1978. MR513816 (80f:47060)

[7] A. Cabot, Inertial gradient-like dynamical system controlled by a stabilizing term, J. Optim. Theory Appl., 120 (2004), 275-303. MR2044898(2004m:90142)

[8] S. Gadat, L. Younes, A stochastic algorithm for feature selection in pattern recognition, $J$. Mach. Learn. Res., 8 (2007), 509-547.

[9] X. Goudou, J. Munier, Asymptotic behavior of solutions of a gradient-like integrodifferential Volterra inclusion, Adv. Math. Sci. Appl., 15 (2005), n 2, 509-525. MR2198574|(2006i:35362)

[10] J. K. Hale, Asymptotic behavior of dissipative systems. Mathematical Surveys and Monographs, 25. American Mathematical Society, Providence, RI, 1988. MR941371 (89g:58059)

[11] A. Haraux, Systèmes dynamiques dissipatifs et applications. Recherches en Mathématiques Appliquées 17, Masson, Paris, 1991. MR 1084372 (92b:35002)

[12] A. Haraux, M. A. Jendoubi, Convergence of solutions of second-order gradient-like systems with analytic nonlinearities, J. Differential Equations, 144 (1998), nº 2, 313-320. MR.1616968 (99a:35182)

[13] M. Hirsch, S. Smale, Differential Equations, Dynamical Systems and Linear Algebra. Academic Press, New York-London, 1974. MR0486784 (58:6484)

[14] S. Maier-Paape, Convergence for radially symmetric solutions of quasilinear elliptic equations is generic, Math. Annalen, 311 (1998), 177-197. MR.1624291 (99g:34011)

[15] J.W. Miles, On a Nonlinear Bessel Equation, SIAM J. Appl. Math., 42 (1982), 109-112. MR646752(83d:65231)

[16] Wei-Ming Ni, Qualitative properties of solutions to elliptic problems. Stationary partial differential equations. In: Handbook of Differential Equations Vol. I, 157-233, North-Holland, Amsterdam, 2004. MR2103689 (2005k:35138)

[17] H. Robbins, S. Monro, A stochastic approximation method, Ann. Math. Stat., 22 (1951), 400-407. MR0042668(13:144j) 
[18] R.T. Rockafellar, Convex Analysis. Princeton University Press, Princeton, 1970. MR0274683 $(43: 445)$

[19] L. Thibault, Sequential convex subdifferential calculus and sequential Lagrange multipliers, SIAM J. on Control and Optimization, 35 (1997), $\mathrm{n}^{\circ}$ 4, 1434-1444. MR/1453305(98f:49020)

Département de Mathématiques, Université Montpellier II, CC 051, Place Eugène Bataillon, 34095 Montpellier Cedex 5, France

E-mail address: acabot@math.univ-montp2.fr

Department of Mathematics, Georgetown University, Box 571233, Washington, DC 20057

E-mail address: engler@georgetown.edu

Laboratoire de Statistique et Probabilités, Université Paul Sabatier, 31062 Toulouse Cedex 9, France

E-mail address: Sebastien.Gadat@math.ups-tlse.fr 